\title{
Adipose Collagen Fragment: an Injectable Sustained-release Collagen Scaffold of Adipokines for Preventing Skin Photoaging in Mice
}

\section{Xiaoxuan Jin}

Southern Medical University Nanfang Hospital

\section{Yuchen Zhang}

Southern Medical University Nanfang Hospital

\section{Xiangdong Zhang}

Southern Medical University Nanfang Hospital

Yibao Li

Southern Medical University Nanfang Hospital

\section{Mimi Xu}

Southern Medical University Nanfang Hospital

\section{Kaiyang Liu}

Southern Medical University Nanfang Hospital

Jiangjiang Ru

Southern Medical University Nanfang Hospital

\section{Chijuan Ma}

Southern Medical University Nanfang Hospital

Yao Yao

Southern Medical University Nanfang Hospital

Yunfan He

Southern Medical University Nanfang Hospital

Jianhua Gao ( $\boldsymbol{\sigma}$ doctorgaojianhua@outlook.com )

Southern Medical University Nanfang Hospital

\section{Research}

Keywords: Skin photoaging, adipokines, extracellular matrix, sustained-release, skin filling, adiposederived product, adipose collagen concentrate

Posted Date: June 7th, 2021

DOI: https://doi.org/10.21203/rs.3.rs-571555/v1 
License: (c) (i) This work is licensed under a Creative Commons Attribution 4.0 International License. Read Full License 


\section{Abstract}

Objective: Ultraviolet A (UVA) radiation is the major contributor to skin photoaging, associated with increased collagen degradation and reactive oxygen species expression. Adipokines have been proven as promising therapeutic agents for skin photoaging. However, adipokine therapy is generally limited by the short in vivo release duration and biological instability. Therefore, developing a treatment that provides a sustained release of adipokines and enhanced therapeutic effects is desirable.

Methods: Adipose collagen fragment (ACF) was prepared from lipoaspirate and characterized. The injectability, collagen components, and adipokine release pattern of ACF were identified in vitro. Then, we evaluated its therapeutic efficacy by injecting ACF and phosphate-buffered saline, as a control, into the dermis of photoaging nude mice and harvesting skin samples at weeks 1, 2, and 4 after treatment for assessment. The collagen synthesis and collagen degradation in ACF implants were evaluated by immune staining. Dermal thickness, fibroblast expression, collagen synthesis, reactive oxygen species level, antioxidase expression, capillary density, and apoptotic cell number were evaluated by histological assessment, immune staining, and polymerase chain reaction in the skin samples.

Results: ACF is the concentrated adipose extracellular matrix collagen fragment without viable cells and can be injected through fine needles. ACF undergoes collagen degradation and promotes neocollagen synthesis in ACF implants. Meanwhile, ACF serves as a sustained-release system of adipokines and exhibits a significantly higher therapeutic effect on mouse skin photoaging compared to controls. ACF increases the dermal thickness, improves fibroblast expression and promotes collagen synthesis. ACF treatment of UVA-irradiated skin reduces reactive oxygen species expression, decreases the number of apoptotic cells, improves capillary density and promotes the expression of antioxidase superoxide dismutase-1, catalase and glutathione peroxidase-1 by sustainedly releasing adipokines.

Conclusions: ACF is an adipokines-enriched, sustained-release extracellular matrix collagen scaffold that can prevent UVA-induced skin photoaging in mice. ACF may serve as a novel autologous skin filler for skin rejuvenation applications in the clinic.

\section{Introduction}

Photoaging attributed to chronic sun exposure (ultraviolet A [UVA] radiation) is a major contributor to skin aging ${ }^{[1-4]}$. UVA exposure increases reactive oxygen species (ROS) production through oxidative metabolism in skin cells ${ }^{[5,6]}$. ROS expression is thought to be a key mediator of fibroblast viability by introducing intracellular DNA damage and protein inactivation ${ }^{[7]}$. Impairments in dermal fibroblasts result in decreased collagen production and remodeling, leading to thin, saggy, and structurally weakened skin $[8,9]$. Therefore, more effective strategies are needed to reduce oxidative stress levels and attenuate UVAinduced cell death to prevent skin photoaging. 
The topical application of antioxidants and stem cells has been proven to be effective in preventing skin photoaging. However, their use in clinics has been limited by poor permeability, healthcare regulatory issues, poor survival of administered cells, and the risk of biological contamination ${ }^{[10-12]}$. The mechanisms underlying stem cell therapy have been largely attributed to cellular paracrine cytokines ${ }^{[10-}$ ${ }^{12]}$. These cytokines act on skin dermal cells to improve skin quality and resist skin aging ${ }^{[16]}$. Recently, studies reported that subcutaneous adipose tissue, located beneath the dermal layer, is closely involved in regulating skin elasticity and contributes to skin physiology ${ }^{[17,18]}$. Importantly, subcutaneous adipose tissue influences dermal conditions through the secretion of various bioactive substances, termed adipokines ${ }^{[19,20]}$. Thus, adipokines appear to be ideal therapeutic agents for preventing skin photoaging. Recently, our research team extracted adipokines, namely adipose liquid extract, from adipose tissue using a purely mechanical method ${ }^{[21-24]}$. Adipose liquid extract contains 1,742 bioactive components and has been found to have a therapeutic effect on wound healing and skin aging by improving angiogenesis, cell viability, collagen synthesis, and attenuating oxidative stress. Nevertheless, given that skin aging is an inevitable and continuous physiological process ${ }^{[25,26]}$, and that the in vivo release duration and biological stability of adipokines are unsatisfactory, a vehicle that can provide a sustained prolonged release as well as maintaining high stability of adipokines with enhanced therapeutic efficacy is desirable.

Adipose tissue is mainly composed of mature adipocytes, stromal vascular fraction, and the extracellular matrix (ECM) ${ }^{[27]}$. The ECM provides natural binding domains to store adipokines secreted by stromal vascular fraction (SVF) cells ${ }^{[28]}$. These adipokines are generally released from ECM scaffolds when exposed to the appropriate stimuli ${ }^{22-32]}$. Therefore, we hypothesized that adipose-derived ECM components could be extracted and serve as a sustained-release scaffold of adipokines for the treatment of skin photoaging.

To verify this hypothesis, we developed a novel mechanical processing technique to extract adipose tissue-derived ECM components, named the "adipose collagen fragment" (ACF). The macrography, injectability, and protein components in ACF were evaluated, and the sustained-release properties of ACF were measured in vitro. Moreover, the therapeutic effect of ACF on UVA-induced photoaging in mice was evaluated using phosphate buffered saline (PBS) as the control.

\section{Methods}

\section{Animals and Skin Photoaging Model}

All experiments were approved by the Nanfang Hospital Animal Ethics Committee Laboratory and were conducted according to the National Health and Medical Research Council of China guidelines. In total, 18 six-week-old female BALB/c nude mice were obtained from the Southern Medical University and maintained in a regulated environment $\left(22 \pm 2^{\circ} \mathrm{C}\right)$ with a $12 \mathrm{~h}$ light/dark cycle at the Animal Experiment Center of Nanfang Hospital, and were fed according to the specific pathogen-free animal criteria. A mouse model of photoaging was used as previously described ${ }^{[33]}$. Briefly, 40 W UVA tubes (wavelength 
range: 350 to $460 \mathrm{~nm} ; 13 \times 10^{2} \mu \mathrm{W} / \mathrm{cm}^{2}$; Sigma-Aldrich, Shanghai, China) were used to irradiate the dorsal skin. The mice were irradiated for two weeks, once daily, for five days a week. We followed an irradiation approach in which the duration of UVA irradiation was $1 \mathrm{~h}$ on day 1 , after which the duration of UVA irradiation was increased by $1 \mathrm{~h}$ per day up to $5 \mathrm{~h}$. The mice were then irradiated for $5 \mathrm{~h}$ daily from days 8-12. The total irradiation intensity of the UVA was approximately $172 \mathrm{~J} / \mathrm{cm}^{2}$.

\section{Human Adipose Tissues Harvest and ACF preparation}

Human adipose tissues in the abdominal regions were obtained from female individuals who underwent liposuction in the Department of Plastic and Reconstruction Surgery, Nanfang Hospital. All patients provided written informed consent. ACF was prepared from fresh lipoaspirates. First, the lipoaspirate was centrifuged at $1,200 \times \mathrm{g}$ for $3 \mathrm{~min}$ to generate Coleman fat. After washing twice with sterilized saline and homogenizing for $60 \mathrm{~s}$ in an ACF extractor (Specially made; Shanghai Tiangong Instruments Co., Ltd., Shanghai, People's Republic of China), the fat suspension was transferred into $20 \mathrm{~mL}$ syringes and then filtered consecutively using unidirectional filters containing a sterilized round stainless-steel filter screen with a $0.25-\mathrm{mm}$ or $0.15-\mathrm{mm}$ sized mesh (Additional file 1). Then, the fat suspension was centrifuged at $3000 \times \mathrm{g}$ for $3 \mathrm{~min}$, and the solid portion at the bottom was collected as ACF (Figure. 1; Additional file 2).

\section{Macrography Evaluation and collagen component analysis of ACF}

ACF was injected through 31-gauge needles to verify the injectability of ACF. For histological evaluation, ACF was loaded onto a glass slide and observed under a light microscope. ACF and standard Coleman fat samples were fixed with $4 \%$ paraformaldehyde, embedded in paraffin, and sliced into $5-\mu \mathrm{m}$ thick sections. Masson staining was performed to assess collagen components. Immunohistochemical staining was performed using antibodies against COL I (ab34710, Abcam, Cambridge, UK), COL IV (ab6586, Abcam, Cambridge, UK), and laminin (ab11575, Abcam, Cambridge, UK).

\section{Live/Dead Cell Staining and Explant Culture}

The cell viability of Coleman fat and ACF was evaluated using live/dead staining. Briefly, $1 \mathrm{~mL}$ of PBS supplemented with $2 \mu \mathrm{L}$ of Calcein-AM $(1 \mathrm{mg} / \mathrm{mL}$ ) (AnaSpec, Fremont, Calif.) and $2 \mu \mathrm{L}$ of DAPI (1 $\mathrm{mg} / \mathrm{mL}$ ) (Sigma-Aldrich, St. Louis, MO, USA) was added to $1 \mathrm{~mL}$ of Coleman fat or ACF samples. After incubating for $10 \mathrm{~min}$ at $37^{\circ} \mathrm{C}$, the samples were observed under a fluorescence microscope (BX 51, Olympus, Japan).

The explant culture was performed according to a standard protocol ${ }^{[34]}$. Briefly, the Coleman fat and ACF were evenly distributed on the surface of $100 \mathrm{~mm}$ culture dishes $(1 \mathrm{~mL} /$ dish). The samples were cultured in complete growth medium (HUXMD-90011, Cyagen, China) at $37^{\circ} \mathrm{C}$ with $5 \%$ humidified $\mathrm{CO}^{2}$ for five days. Then, the outgrown cells from explants were observed under a light microscope (GX53, Olympus, Japan). 


\section{Proteomic mass spectrometry and bioinformatics analysis of ACF}

The Trypsin was used to digest the ACF samples for LC-MS/MS analysis according to a previous protocol [35]. LC-MS/MS analysis was performed using a nano LC system (DIONEX ThermoFisher Scientific). The tryptic peptides were fractionated and subjected to reversed-phase liquid chromatography. Peptide samples were separated on a self-packed column (ThermoFisher Scientific, Acclaim PepMap RSLC $50 \mu m$ $\times 15 \mathrm{~cm}$, nanoviper, P/N164943) at a flow rate of $300 \mathrm{~nL} / \mathrm{min}$, according to a previous protocol ${ }^{[36]}$. Labelfree quantification (LFQ) was performed as previously described ${ }^{[37]}$. The resulting data for LFQ were processed using the MaxQuant program (version 1.5.3.1), and Andromeda was used to match MS/MS spectra as a database search engine according to the human database ${ }^{[38]}$.

Gene Ontology (GO) database (http://www.geneontology.org) was used to perform functional annotation analysis, including cell component, molecular function, and biological process ${ }^{[39]}$. Protein location information of all identified proteins in ACF was obtained using Ingenuity Pathway Analysis analysis through a web database (https://www.qiagenbioinformatics.com). Proteins participated in diverse collagen biological process were identified. Collagen types of ACF were classified according to the protein identification list. Proteins related to angiogenesis, antioxidant ability, cell proliferation, and apoptosis were identified and summarized.

\section{In vitro release of adipokines from ACF}

The ACF were cultured with Dulbecco's modified Eagle's medium (ThermoFisher Scientific, Foster City, CA, USA) in $100 \mathrm{~mm}$ plate dishes and maintained at $37^{\circ} \mathrm{C}$ with $5 \% \mathrm{CO}_{2}$. DMEM $(9 \mathrm{~mL})$ was added to $1 \mathrm{~mL}$ of ACF to each dish. The culture medium was replaced on days 2 and 6 and collected on days 1,3 and 7 . Protein concentrations of the culture media were measured using a BCA protein assay kit to determine the total amount of released adipokines. To identify the amount of released fibroblast growth factor (FGF), vascular endothelial growth factor (VEGF), and adiponectin, protein quantification of the culture medium was carried out using ELISA kits (R\&D Systems, Minneapolis, MN, USA).

\section{ACF labeling and animal experiments}

The collagen component of ACF was labeled according to a previously described protocol ${ }^{[40]}$. Briefly, 3 $\mathrm{mL}$ of ACF were incubated with $100 \mu \mathrm{M}$ Alexa Fluor 647 NHS Ester (A20006, ThermoFisher Scientific, Foster City, CA, USA) for $1 \mathrm{~h}$ at $25^{\circ} \mathrm{C}$, followed by three washes with PBS.

The ACF were injected into the mouse skin of the left dorsa, with a PBS injection into the right side serving as the control ( 9 spots/side, $0.01 \mathrm{ml} / \mathrm{spot}$ ). Animals were sacrificed, and skin samples were harvested for further analyses on weeks 1,2 , and 4 postoperatively ( $n=6 /$ time point). One-half of the skin samples were fixed with paraformaldehyde, and the other half of the skin samples were immediately stored at $-80^{\circ} \mathrm{C}$ for further experiments. 


\section{Histological assessment and immune staining of skin samples}

Mouse skin samples were embedded in paraffin and sliced into $5-\mu \mathrm{m}$ thick sections. Masson staining was performed to assess collagen content in the dermis and ACF implants. The expression of fibroblasts and newly formed collagen in the dermis and ACF implants was evaluated using antibodies against vimentin (ab92547, Abcam, Cambridge, UK) and procollagen (ab64409, Abcam, Cambridge, UK), followed by secondary antibodies. Angiogenesis was evaluated using antibodies against CD31 antibody (1:25, ab28364, Abcam, Cambridge, UK), followed by secondary antibodies. A TUNEL staining kit (Roche Molecular Biochemicals, Mannheim, Germany) was used for detecting apoptotic cells in skin samples according to the manufacturer's protocol. ROS levels were measured by the DCFH2-DA staining kit (S0063, Beyotime, China). The expression of antioxidant enzymes in skin samples was evaluated using antibodies against superoxide dismutase-1 (SOD-1; PB0453, Boster, China), catalase (PB0971, Boster, China) and glutathione peroxidase-1(GPX-1; PB9203, Boster, China), followed by secondary antibodies. The sections were then observed under a microscope (BX 51, Olympus, Japan; LSM 980, Zeiss Axioscope, Oberkochen, Germany). Qualification analyses of dermal thickness, fibroblasts, neocollagen, capillary density, apoptotic cells, ROS levels, and antioxidant enzymes were assessed using Image $\mathrm{J}$ software.

\section{Quantitative reverse transcription PCR}

Total RNA was extracted and reverse transcribed into cDNA using a reverse transcription kit (ThermoFisher Scientific, Foster City, CA, USA). cDNA was amplified and taken as a template. qRT-PCR was performed on QuantStudio Real-Time PCR Systems (Applied Biosystems, USA). The relative mRNA expression was calculated by the $2-\Delta \triangle C$ t method, with $\beta$-actin as the reference gene. Primer sequences were used as follows: SOD-1, forward 5-GGTTCCACGTCCATCAGT-3 and reverse 5ACATTGCCCAGGTCTCC-3; catalase, forward 5-GAAGGCTTGCTCAGGAAGAT-3 and reverse 5TGCCAACTGGTATAAGAGGGTA-3; GPX-1, forward 5-ATCAGTTCGGACACCAGGA-3 and reverse 5TCTCACCATTCACTTCGCA-3; $\beta$-actin, forward 5-GAGGTATCCTGACCCTGAAGTA-3 and reverse 5CACACGCAGCTCATTGTAGA-3'.

\section{Statistical Analysis}

All data are expressed as mean \pm SD. Statistical analyses were performed using SPSS software (version 26.0; IBM Corp., Armonk, NY, USA). An unpaired t-test was used to compare the two groups at a single time point. Statistical significance was set at $p<0.05$.

\section{Results}

\section{ACF is an adipose ECM fragment without viable cells}

Figure 2a shows macroscopic observations of Coleman fat and ACF. ACF is a pinkish-white, homogeneous, and viscous substance with a smooth texture. ACF can be injected through 31-gauge needles (Fig. 2b). Figure 3a shows that collagen was expressed in the entire field of view in ACF, while the 
collagen components in Coleman fat can only be detected in the surrounding adipocytes. Figure $3 \mathrm{~b}$ shows that ACF is composed of collagen fragments. COL I, COL IV, and laminin expression in ACF can be detected throughout the entire field of view, while these collagens can only be observed surrounding adipocytes in Coleman fat $(\mathrm{p}<0.05)$ (Fig. 4a,b). Massive dead cells (red) were observed in the ACF, while only a few dead cells (red) were observed in Coleman fat (Fig. 5). No outgrown cells were observed in the ACF explant culture assays; nevertheless, many fibroblastic-like cells were detected outgrown from Coleman fat explants.

\section{ACF is an adipokines sustained-release collagen scaffold}

A total of 2,555 proteins were quantified. Proteins were classified by GO annotation based on three categories: cellular components, molecular functions, and biological processes (Fig. 6d). The three most abundant classes of biological processes were cellular processes, biological regulation, and metabolic processes. In addition, the molecular function analysis showed that most of the quantified proteins in ACF were classified in the classes of binding, catalytic activity, and molecular function regulators. For the result of the cellular component, the majority of ACF proteins were in the cell, organelle, and proteincontaining complex GO category. Figure 6a shows that most of the quantified proteins of ACF were from the plasma membrane, followed by the nucleus, and finally from the extracellular space and cytoplasm. Figure $6 \mathrm{~b}$ shows that most proteins participated in the collagen metabolic process and collagen fibril organization, followed by the collagen biosynthetic process and collagen catabolic process. Figure 6c describes that the most abundant collagen types were type I, type VI, and type IV. Functional annotation revealed that a great variety of proteins are involved in angiogenesis, antioxidation, cell proliferation, and apoptosis (Table 1).

Figure 7 shows the release curves of adipokines of ACF in vitro. Adipokines release increased over time from days $1-3$, and reached a maximum value on day 3 . Adipokines concentrations decreased over time in the subsequent four days (Fig. 7a). The amount of FGF released from ACF increased over time from days $1-3$, and the FGF release reached a maximum value on day 3 (Fig. $7 \mathrm{~b}$ ). The release concentration of FGF decreased over the following four days. VEGF release reached a maximum value on day one and then decreased from days 1-3 (Fig. 7c). The released VEGF displayed a plateaued release between days 3-7. Over the following seven days, the concentration of VEGF decreased over time. ACF released adiponectin rapidly from days $1-3$, and adiponectin release reached a maximum value on day 3 (Fig. $7 \mathrm{~d}$ ). Adiponectin concentrations decreased over time in the subsequent four days.

\section{ACF undergoes collagen degradation and promotes neocollagen synthesis in ACF implants}

Figure 8a shows the phenomenon of collagen degradation in ACF in vivo. ACF underwent a slow degradation process from weeks $1-2(p>0.05)$, followed by a fast degradation process from weeks $2-4$ $(p<0.05)$ (Fig. 8b). Procollagen expression was detected in ACF implants at all time points. In ACF 
implants, the semi-quantification analysis showed that procollagen expression was maximal at week one and then decreased from weeks $2-4(p<0.05)$.

ACF enhances the dermal thickness in the mouse dermis and promotes fibroblast expression in ACF implants and the mouse dermis.

Masson staining showed a significantly higher level of dermal thickness and collagen expression in the ACF-treated group at week 1, compared to the control group (Fig. 9a). Semi-quantification analysis demonstrated that the dermal thickness of the ACF-treated group was higher than that in the control group at each time point $(p<0.05)$ (Fig. 9b). Immunohistochemical staining showed that fibroblasts were observed in mouse skin and ACF implants at week 1 (Fig. 9a). Semi-quantification analysis demonstrated that the expression level of fibroblasts in the dermis of the ACF-treated group was maximal at week 1 and decreased from weeks $2-4$, and was higher than that in the control group at each time point $(p<0.05)$ (Fig. 9b).

\section{ACF reduces ROS production and induces antioxidant proteins expression in the mouse dermis}

Immunofluorescence staining showed that ROS production in the skin tissue was detected in both groups at all time points (Fig. 10a). Semi-quantification revealed that a higher level of ROS was observed in the control group than in the ACF-treated group at each time point $(P<0.05)$ (Fig. 10b).

Immunohistochemical staining showed that the expression of antioxidant enzyme SOD-1, catalase, and GPX-1 was detected in both the ACF-treated and control groups at each time point (Fig. 11a, 12a, 13a). PCR evaluated the relative antioxidative gene expression of SOD-1, catalase, and GPX-1 in the mouse skin. The expression of catalase and SOD-1 was higher in the ACF-treated group than in the control group at weeks 2-4 ( $<$ 0.05) (Fig. 11b, 12b). The higher expression of GPX-1 was detected in the ACF-treated than that in control groups at week $4(p<0.05)$ (Fig. 13b).

\section{ACF promotes angiogenesis and reduces cell apoptosis in the mouse dermis}

Immunofluorescence staining showed that the expression level of neo-vessels was maximal at week 1 in both groups (Fig. 14a). Semi-quantification of CD31 + neo-vessels revealed that the number of CD31+ vessels was significantly higher in the ACF-treated group than in the control group at all time points $(\mathrm{p}<$ 0.05) (Fig. 14b). TUNEL-positive cells were observed in both groups at all time points (Fig. 15a). Semiquantification analysis demonstrated that more TUNEL-positive cells were observed in the control group than in the ACF-treated group at each time point $(p<0.05)($ Fig. 15b).

\section{Discussion}

In this study, an injectable, adipokine-enriched, collagen concentrate (ACF) was produced from lipoaspirate using a fast and pure mechanical method. ACF experienced a gradual degradation process in 
vivo, and exhibited a sustained release of adipokines in vitro. ACF prevents skin photoaging in mice by stimulating collagen synthesis, increasing fibroblast expression, inducing capillary formation, promoting antioxidant action, and attenuating cell apoptosis.

Sustain release systems are considered a superior approach for enhancing growth factor-based therapies with a short release duration, which requires repeated dosing ${ }^{[41]}$. In this study, immunohistochemistry staining and a mass spectrometry analysis showed that ACF is an adipose ECM concentrate containing a large number of adipokines. The ECM is a highly specialized three-dimensional network consisting of collagen scaffolds and scaffold-bound bioactive components ${ }^{[42]}$. ECM binding sites, such as GAGs and proteoglycans, can bind to and sequester bioactive components ${ }^{[43]}$ as well as maintain their activity and stability ${ }^{[44]}$. A release profile analysis showed a sustained release of adipokines from ACF in vitro, and our fluorescence tracing results indicated a gradual degradation process of ACF in vivo. Notably, the ECM serves as a natural reservoir of growth factors and releases them when exposed to the appropriate stimuli $[45,46]$. The mechanisms that regulate the release of ECM-bound growth factors are complex, including binding affinity, conformational changes, and degradation of the ECM ${ }^{[47,48]}$. Therefore, ACF may be considered as a sustained release system of adipokines and thus partly explain the therapeutic effects of ACF. In the past decade, several artificial sustained-release scaffolds made of synthetic materials have been developed to prevent skin photoaging ${ }^{[49-51]}$. Although artificial scaffolds can prolong the effective drug release duration, hydrophilia deficiencies and a lack of bioactivity may compromise the ability of artificial scaffolds to facilitate material-host interactions. Furthermore, their degradation products may generate an acidic environment conducive to an adverse inflammatory response and considerable cytotoxicity $[52,53]$.

Skin photoaging is characterized by a combination of histological findings, including decreased dermis thickness, increased collagen fragmentation, increased oxidative stress, and increased inflammatory reactions ${ }^{[25]}$. Therefore, a comprehensive therapeutic strategy that ameliorates multiple interrelated manifestations of photoaging is highly desirable. GO analysis showed that the adipokines found in ACF, such as adiponectin, VEGF, FGF, transforming growth factor- $\beta$, endothelial growth factor and hepatocyte growth factor, are involved in various biological processes. The therapeutic effects of these adipokines have been investigated in several studies. For instance, adiponectin exerts an antioxidant effect on fibroblasts by decreasing basal matrix metalloproteinase- 1 expression and inducing the expression of procollagen ${ }^{[19]}$. VEGF-B protects retinal cells against oxidative stress and rescues retinal degeneration by upregulating the antioxidative genes GPX1 and SOD1 ${ }^{[54]}$. Hepatocyte growth factor protects mesenchymal stem cells against $\mathrm{H}_{2} \mathrm{O}_{2}$-induced apoptosis by decreasing the phosphorylation of extracellular signal-regulated kinases and p38 ${ }^{[54]}$. In this study, the therapeutic effects of ACF on photoaging are likely due to an attenuation of oxidative stress levels, an improvement in fibroblast viability, stimulation of collagen synthesis, inhibition of apoptosis, and stimulation of blood vessel formation. Importantly, the comprehensive therapeutic effects of ACF may be explained by the presence 
of a diversity of adipokines. Nevertheless, the mechanisms underlying adipokines therapeutic effects in ACF need to be investigated in the future.

In the past decade, several adipose-derived products, such as microfat and SVF-gel, were developed and considered to exert a therapeutic effect on the skin ${ }^{[56,57]}$. However, limited by the particle size, intradermal injection of these adipose derivatives may be largely limited ${ }^{[58-60]}$. In addition, the fat particles and lipids contained in these adipose derivatives may cause yellowish discoloration and nodules after a superficial subcutaneous injection. Compared with adipose derivative therapies and stem cell-based therapy, the advantages of using ACF can be summed up as follows: (1) ACF is adipose collagen fragments without fat particles and lipids, and therefore does not cause yellowish discoloration and nodules after intradermal injection. (2) ACF is a viable cell-free production and therefore can overcome the challenges of stem cell-based therapies, for example, healthcare regulatory issues, poor survival of administered cells, and the risk of biological contamination. (3) ACF is obtained from lipoaspirate through a fast and pure mechanical method without chemical and biological contamination, largely reducing any potential safety hazards. (4) Prepared using simple and rapid methods, ACF can be obtained in operating rooms during surgery, largely reducing the human and economic burden. (5) Current strategies for preventing skin photoaging are mainly focused on stimulating neocollagen synthesis in the dermis ${ }^{[61-63]}$. In comparison, $\mathrm{ACF}$, as the adipose-derived collagen concentrate, can directly replenish dermal collagen components and simultaneously stimulate neocollagen synthesis in the dermis. (6) Skin aging is an inevitable and continuous physiological process ${ }^{[25]}$ that requires repeated treatments to maintain the desired therapeutic effect. Lyophilization is a convenient, safe, and cost-effective method for preserving biological components ${ }^{[64]}$. Lyophilized ACF for repeated future use will be investigated in the following studies. In spite of these advantages, there are still some limitations that remain to be improved. First, the optimum therapeutic concentration and dose of ACF remains unclear and should be investigated. Second, the duration of in vivo degradation of ACF needs to be assessed to help determine the optimal therapeutic schedule. Third, the therapeutic effects of ACF on skin photoaging in clinical settings needs to be verified. Fourth, as an adipokine reservoir, the therapeutic effects of ACF on skin disorders, such as melasma and atopic dermatitis, are unknown and should be determined. Fifth, the adipokines release pattern of ACF was only studied using an in vitro experiment due to the limitations and the in vivo release pattern is worthy of future investigation.

\section{Conclusions}

ACF is an adipokines-enriched, sustained-release ECM collagen scaffold that can prevent UVA-induced skin photoaging in mice model through enhancing angiogenesis, antioxidant abilities, antiapoptotic activities, and collagen synthesis in mice. ACF may serve as a novel autologous skin filler for skin rejuvenation applications in the clinic.

\section{Abbreviations}


ACF: Adipose collage fragments

ECM: Extracellular matrix

UVA: Ultraviolet A

ROS: Reactive oxygen species

SVF: Stromal vascular fraction

PBS: Phosphate buffered saline

LFQ: Label-free quantification

GO: Gene ontology

FGF: Fibroblast growth factor

VEGF: Vascular endothelial growth factor

GPX-1: Glutathione peroxidase 1

SOD-1: Superoxide dismutase-1

\section{Declarations}

Ethics approval and consent to participate

All animal experiments were approved by Nanfang Hospital Institutional Animal Care and Use Committee and conducted according to the guidelines of the National Health and Medical Research Council (China).

\section{Consent for publication}

Not applicable

\section{Availability of data and materials}

All data generated or analyzed in this study are included in this article.

\section{Competing interests}

The authors declare that they have no competing interests

\section{Funding}

This work was supported by the National Nature Science Foundation of China $(81772101,81801933$, 81871573), the Natural Science Foundation of Guangdong Province of China (2017A030313900), and 
the Administrator Foundation of Nanfang Hospital (2016Z010, 2017C008).

\section{Authors' contributions}

HJG contributed to the conception and design. FYH and YY contributed to the design and helped with manuscript writing. XXJ contributed to the data analysis and manuscript writing. CYZ helped with the in vivo experiments. DXZ helped with the in vitro experiments and data analysis. $B Y L, M M X, Y K L, J J R$ and JCM helped with the interpretation and data collection. All authors read and approved the final manuscript.

\section{Acknowledgements}

The authors thank the Institute of Research Center of Clinical Medicine, Nanfang Hospital for providing comprehensive experimental services.

\section{References}

[1] Wang F, Smith N R, Tran B A, et al. Dermal damage promoted by repeated low-level UV-A1 exposure despite tanning response in human skin[J]. JAMA Dermatol, 2014,150(4):401-406.

[2] Liu J, Zhang W. The influence of the environment and clothing on human exposure to ultraviolet light[J]. PLoS One, 2015,10(4):e124758.

[3] Gilchrest B A. Photoaging[J]. J Invest Dermatol, 2013,133(E1):E2-E6.

[4] Kammeyer A, Luiten R M. Oxidation events and skin aging[J]. Ageing Res Rev, 2015,21:16-29.

[5] Bosch R, Philips N, Suarez-Perez J A, et al. Mechanisms of Photoaging and Cutaneous Photocarcinogenesis, and Photoprotective Strategies with Phytochemicals[J]. Antioxidants (Basel), 2015,4(2):248-268.

[6] Fabi S, Sundaram $\mathrm{H}$. The potential of topical and injectable growth factors and cytokines for skin rejuvenation[J]. Facial Plast Surg, 2014,30(2):157-171.

[7] Rinnerthaler M, Bischof J, Streubel M K, et al. Oxidative stress in aging human skin[J]. Biomolecules, 2015,5(2):545-589.

[8] Purohit T, He T, Qin Z, et al. Smad3-dependent regulation of type I collagen in human dermal fibroblasts: Impact on human skin connective tissue aging[J]. J Dermatol Sci, 2016,83(1):80-83.

[9] Xia W, Quan T, Hammerberg C, et al. A mouse model of skin aging: fragmentation of dermal collagen fibrils and reduced fibroblast spreading due to expression of human matrix metalloproteinase-1[J]. J Dermatol Sci, 2015,78(1):79-82. 
[10] Yeager D G, Lim H W. What's New in Photoprotection: A Review of New Concepts and Controversies[J]. Dermatol Clin, 2019,37(2):149-157.

[11] Masaki H. Role of antioxidants in the skin: anti-aging effects[J]. J Dermatol Sci, 2010,58(2):85-90.

[12] Sato Y, Bando H, Di Piazza M, et al. Tumorigenicity assessment of cell therapy products: The need for global consensus and points to consider[J]. Cytotherapy, 2019,21(11):1095-1111.

[13] Kim W S, Park B S, Sung J H. Protective role of adipose-derived stem cells and their soluble factors in photoaging[J]. Arch Dermatol Res, 2009,301(5):329-336.

[14] Xu X, Wang H Y, Zhang Y, et al. Adipose-derived stem cells cooperate with fractional carbon dioxide laser in antagonizing photoaging: a potential role of Wnt and beta-catenin signaling[J]. Cell Biosci, 2014,4:24.

[15] Altman A M, Abdul K F, Seidensticker M, et al. Human tissue-resident stem cells combined with hyaluronic acid gel provide fibrovascular-integrated soft-tissue augmentation in a murine photoaged skin model[J]. Plast Reconstr Surg, 2010,125(1):63-73.

[16] Hodgkinson C P, Bareja A, Gomez J A, et al. Emerging Concepts in Paracrine Mechanisms in Regenerative Cardiovascular Medicine and Biology[J]. Circ Res, 2016,118(1):95-107.

[17] Ezure T, Amano S. Influence of subcutaneous adipose tissue mass on dermal elasticity and sagging severity in lower cheek[J]. Skin Res Technol, 2010,16(3):332-338.

[18] Lee K E, Nho Y H, Yun S K, et al. Caviar Extract and Its Constituent DHA Inhibits UVB-Irradiated Skin Aging by Inducing Adiponectin Production[J]. Int J Mol Sci, 2020,21(9).

[19] Kim E J, Kim Y K, Kim M K, et al. UV-induced inhibition of adipokine production in subcutaneous fat aggravates dermal matrix degradation in human skin[J]. Sci Rep, 2016,6:25616.

[20] Halberg N, Wernstedt-Asterholm I, Scherer P E. The adipocyte as an endocrine cell[J]. Endocrinol Metab Clin North Am, 2008,37(3):753-768.

[21] Cai Y, Li J, Jia C, et al. Therapeutic applications of adipose cell-free derivatives: a review[J]. Stem Cell Res Ther, 2020,11(1):312.

[22] He $\mathrm{Y}$, Xia J, Chen $\mathrm{H}$, et al. Human adipose liquid extract induces angiogenesis and adipogenesis: a novel cell-free therapeutic agent[J]. Stem Cell Research \& Therapy, 2019,10(1).

[23] Deng M, Xu Y, Yu Z, et al. Protective Effect of Fat Extract on UVB-Induced Photoaging In Vitro and In Vivo[J]. Oxid Med Cell Longev, 2019,2019:6146942.

[24] Yu Z, Cai Y, Deng M, et al. Fat extract promotes angiogenesis in a murine model of limb ischemia: a novel cell-free therapeutic strategy[J]. Stem Cell Res Ther, 2018,9(1):294. 
[25] Gu Y, Han J, Jiang C, et al. Biomarkers, oxidative stress and autophagy in skin aging[J]. Ageing Res Rev, 2020,59:101036.

[26] Gal S, Pu L. An Update on Cryopreservation of Adipose Tissue[J]. Plast Reconstr Surg, 2020,145(4):1089-1097.

[27] Mori S, Kiuchi S, Ouchi A, et al. Characteristic expression of extracellular matrix in subcutaneous adipose tissue development and adipogenesis; comparison with visceral adipose tissue[J]. Int J Biol Sci, 2014,10(8):825-833.

[28] van Dongen J A, Getova V, Brouwer L A, et al. Adipose tissue-derived extracellular matrix hydrogels as a release platform for secreted paracrine factors[J]. J Tissue Eng Regen Med, 2019,13(6):973-985.

[29] Choi J S, Yang H J, Kim B S, et al. Fabrication of porous extracellular matrix scaffolds from human adipose tissue[J]. Tissue Eng Part C Methods, 2010,16(3):387-396.

[30] Kanematsu A, Yamamoto S, Ozeki M, et al. Collagenous matrices as release carriers of exogenous growth factors[J]. Biomaterials, 2004,25(18):4513-4520.

[31] Hynes R O. The extracellular matrix: not just pretty fibrils[J]. Science, 2009,326(5957):1216-1219.

[32] Bhardwaj T R, Kanwar M, Lal R, et al. Natural gums and modified natural gums as sustained-release carriers[J]. Drug Dev Ind Pharm, 2000,26(10):1025-1038.

[33] Zhou R, Wang M, Zhang X, et al. Therapeutic effect of concentrated growth factor preparation on skin photoaging in a mouse model[J]. J Int Med Res, 2020,48(10):1220762498.

[34] Sineh S K, Razavi A, Hassan Z M, et al. Comparative immunomodulatory properties of mesenchymal stem cells derived from human breast tumor and normal breast adipose tissue[J]. Cancer Immunol Immunother, 2020,69(9):1841-1854.

[35] Wiśniewski J R, Nagaraj N, Zougman A, et al. Brain Phosphoproteome Obtained by a FASP-Based Method Reveals Plasma Membrane Protein Topology[J]. Journal of Proteome Research, 2010,9(6):32803289.

[36] Hauck S M, Dietter J, Kramer R L, et al. Deciphering membrane-associated molecular processes in target tissue of autoimmune uveitis by label-free quantitative mass spectrometry[J]. Mol Cell Proteomics, 2010,9(10):2292-2305.

[37] Luber C A, Cox J, Lauterbach $\mathrm{H}$, et al. Quantitative proteomics reveals subset-specific viral recognition in dendritic cells[J]. Immunity, 2010,32(2):279-289.

[38] Cox J, Neuhauser N, Michalski A, et al. Andromeda: a peptide search engine integrated into the MaxQuant environment[J]. J Proteome Res, 2011,10(4):1794-1805. 
[39] Gotz S, Garcia-Gomez J M, Terol J, et al. High-throughput functional annotation and data mining with the Blast2GO suite[J]. Nucleic Acids Res, 2008,36(10):3420-3435.

[40] Correa-Gallegos D, Jiang D, Christ S, et al. Patch repair of deep wounds by mobilized fascia[J]. Nature, 2019,576(7786):287-292.

[41] Davoodi P, Lee L Y, Xu Q, et al. Drug delivery systems for programmed and on-demand release[J]. Adv Drug Deliv Rev, 2018,132:104-138.

[42] Mecham R P. Overview of extracellular matrix[J]. Curr Protoc Cell Biol, 2012,Chapter 10:10-11.

[43] Witjas F, van den Berg B M, van den Berg C W, et al. Concise Review: The Endothelial Cell Extracellular Matrix Regulates Tissue Homeostasis and Repair[J]. Stem Cells Transl Med, 2019,8(4):375-382.

[44] Sutherland I W. The biofilm matrix-an immobilized but dynamic microbial environment[J]. Trends Microbiol, 2001,9(5):222-227.

[45] Ma Z, Mao C, Jia Y, et al. Extracellular matrix dynamics in vascular remodeling[J]. Am J Physiol Cell Physiol, 2020,319(3):C481-C499.

[46] Martino M M, Briquez P S, Maruyama K, et al. Extracellular matrix-inspired growth factor delivery systems for bone regeneration[J]. Adv Drug Deliv Rev, 2015,94:41-52.

[47] Sternlicht M D, Werb Z. How matrix metalloproteinases regulate cell behavior[J]. Annu Rev Cell Dev Biol, 2001,17:463-516.

[48] Afratis N A, Klepfish M, Karamanos N K, et al. The apparent competitive action of ECM proteases and cross-linking enzymes during fibrosis: Applications to drug discovery[J]. Adv Drug Deliv Rev, 2018,129:415.

[49] Fan $\mathrm{Y}$, Choi $\mathrm{T} \mathrm{H}$, Chung $\mathrm{J} \mathrm{H}$, et al. Hyaluronic acid-cross-linked filler stimulates collagen type 1 and elastic fiber synthesis in skin through the TGF-beta/Smad signaling pathway in a nude mouse model[J]. $J$ Plast Reconstr Aesthet Surg, 2019,72(8):1355-1362.

[50] Garcia-Gonzalez C A, Sampaio D S A, Argemi A, et al. Production of hybrid lipid-based particles loaded with inorganic nanoparticles and active compounds for prolonged topical release[J]. Int J Pharm, 2009,382(1-2):296-304.

[51] de Oliveira M M, Nakamura C V, Auzely-Velty R. Boronate-ester crosslinked hyaluronic acid hydrogels for dihydrocaffeic acid delivery and fibroblasts protection against UVB irradiation[J]. Carbohydr Polym, 2020,247:116845.

[52] George S. Hussey J L D A. Extracellular matrix-based materials for regenerative medicine[J]. Nature Revlews, 2018. 
[53] Wang S, Li R, Xia D, et al. The impact of Zn-doped synthetic polymer materials on bone regeneration: a systematic review[J]. Stem Cell Res Ther, 2021,12(1):123.

[54] Arjunan P, Lin X, Tang Z, et al. VEGF-B is a potent antioxidant[J]. Proceedings of the National Academy of Sciences, 2018,115(41):10351-10356.

[55] Choi Y J, Lee C M, Lee J H, et al. Protective effects of hepatocyte growth factor gene overexpression against hydrogen peroxide-induced apoptosis in mesenchymal stem cells[J]. Environ Toxicol, 2019,34(11):1236-1245.

[56] Xu P, Yu Q, Huang H, et al. Nanofat Increases Dermis Thickness and Neovascularization in Photoaged Nude Mouse Skin[J]. Aesthetic Plast Surg, 2018,42(2):343-351.

[57] Yang Z, Jin S, He Y, et al. Comparison of Microfat, Nanofat and Extracellular Matrix/Stromal Vascular Fraction Gel for Skin Rejuvenation: Basic Research and Clinical Applications[J]. Aesthet Surg J, 2021.

[58] Cohen S R, Hewett S, Ross L, et al. Regenerative Cells For Facial Surgery: Biofilling and Biocontouring[J]. Aesthet Surg J, 2017,37(suppl_3):S16-S32.

[59] Suh A, Pham A, Cress M J, et al. Adipose-derived cellular and cell-derived regenerative therapies in dermatology and aesthetic rejuvenation[J]. Ageing Res Rev, 2019,54:100933.

[60] Tonnard P, Verpaele A, Peeters G, et al. Nanofat grafting: basic research and clinical applications[J]. Plast Reconstr Surg, 2013,132(4):1017-1026.

[61] Lee D H, Oh J H, Chung J H. Glycosaminoglycan and proteoglycan in skin aging[J]. J Dermatol Sci, 2016,83(3):174-181.

[62] Hu S, Li Z, Cores J, et al. Needle-Free Injection of Exosomes Derived from Human Dermal Fibroblast Spheroids Ameliorates Skin Photoaging[J]. ACS Nano, 2019,13(10):11273-11282.

[63] Chung C L, Lawrence I, Hoffman M, et al. Topical rapamycin reduces markers of senescence and aging in human skin: an exploratory, prospective, randomized trial[J]. Geroscience, 2019,41(6):861-869.

[64] Nail S L, Jiang S, Chongprasert S, et al. Fundamentals of freeze-drying[J]. Pharm Biotechnol, 2002,14:281-360.

\section{Tables}

Due to technical limitations, table 1 is only available as a download in the Supplemental Files section.

\section{Figures}




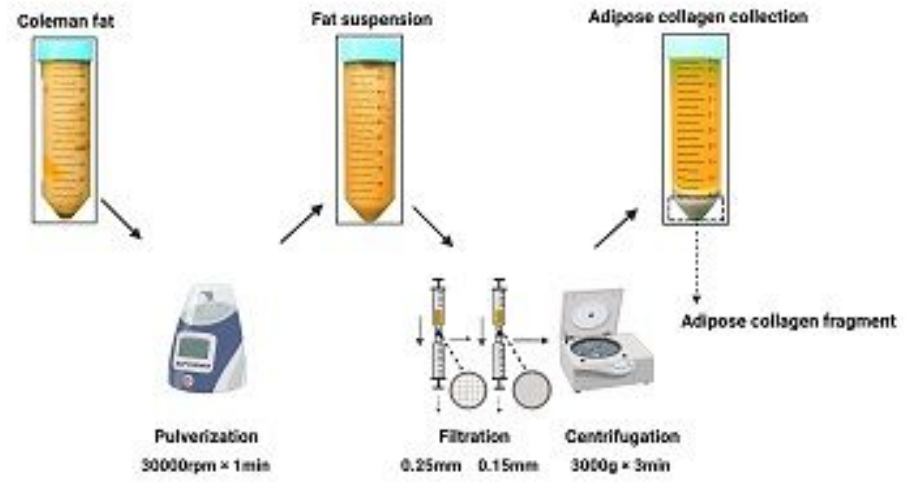

Figure 1

Schematic overview of ACF preparation.

Page 18/29 

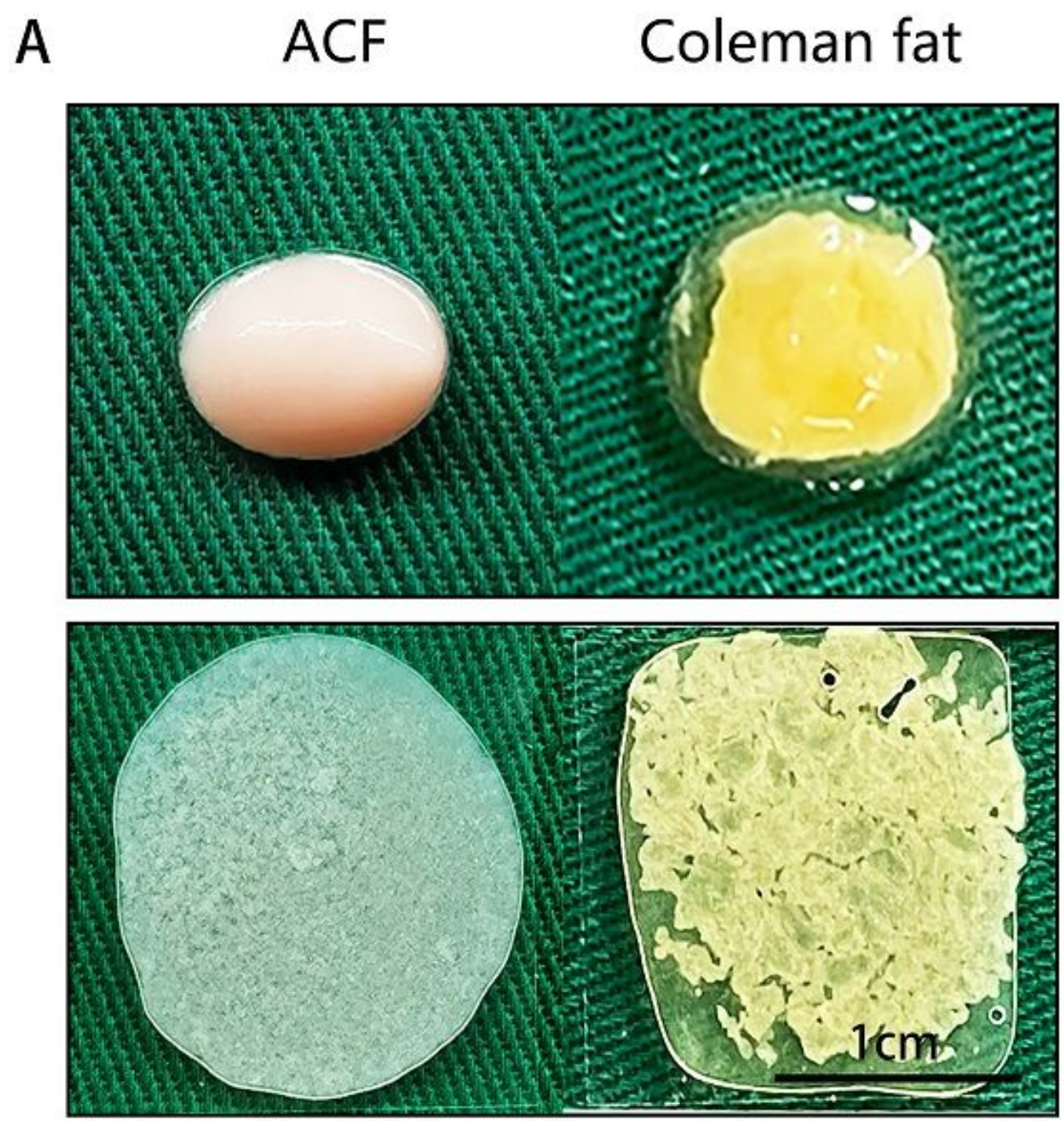

B

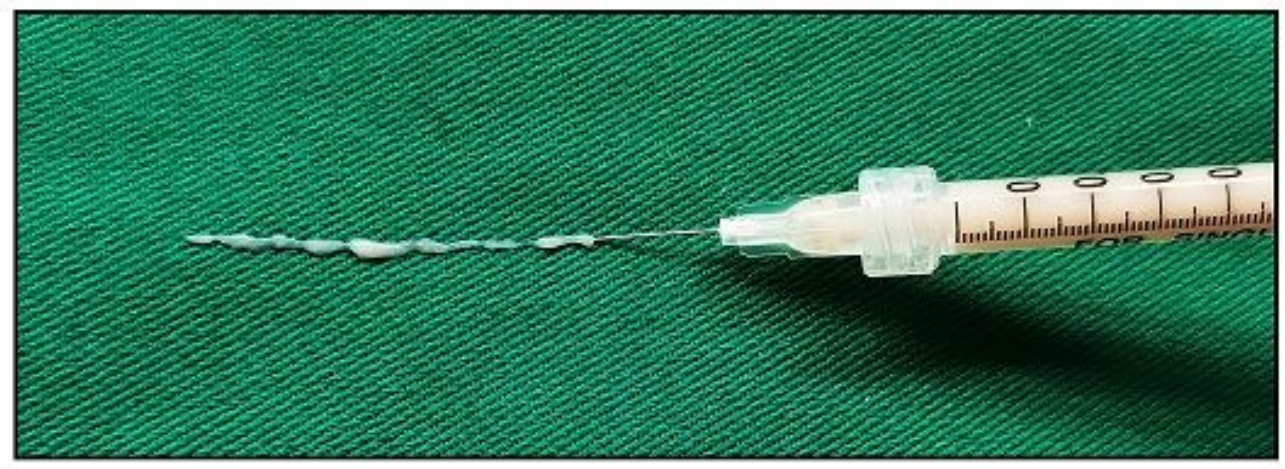

Figure 2

Macroscopic observation and Injectability of ACF. a Macroscopic observation of Coleman fat and ACF. Scale bar $=1 \mathrm{~cm}$. b ACF could be injected through 31-gauge sharp needles. 


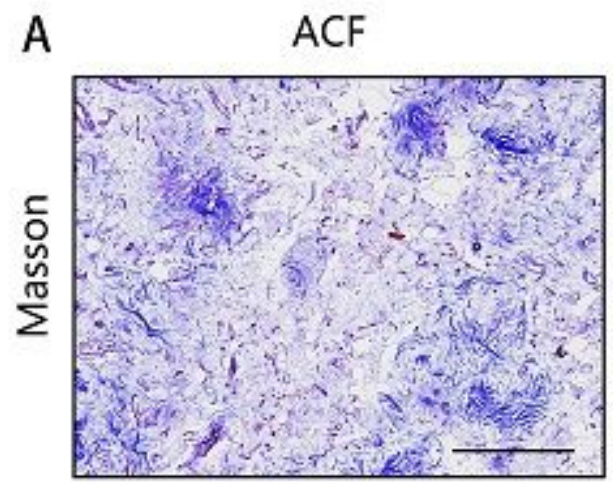

Coleman fat

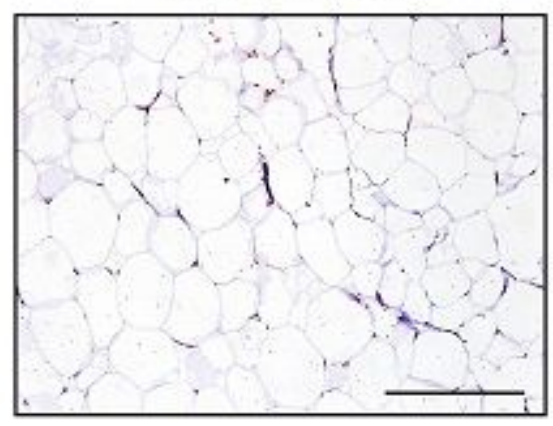

B

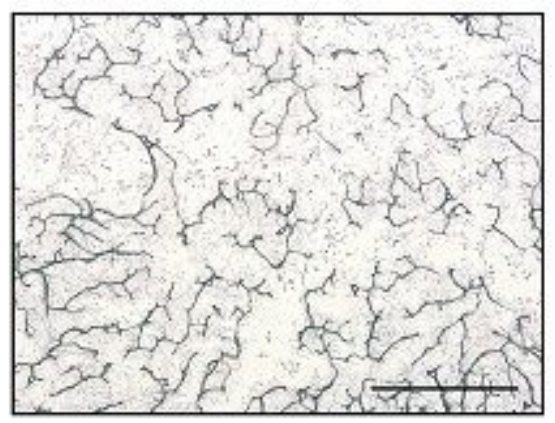

\section{Figure 3}

Histological evaluation of Coleman fat and ACF. a Masson staining of Coleman fat and ACF. Scale bar $=$ $200 \mu \mathrm{m}$. b Scattered, stripe-like collagen fragments in ACF were observed in the bright-field image. Scale bar $=1 \mathrm{~cm}$.

A
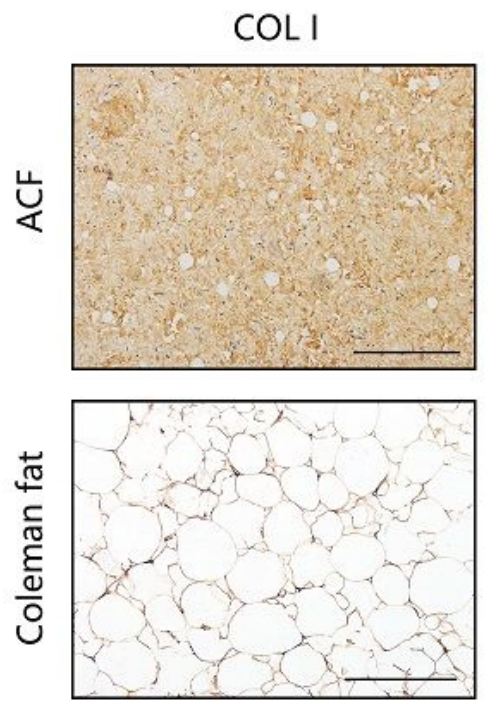

COL IV
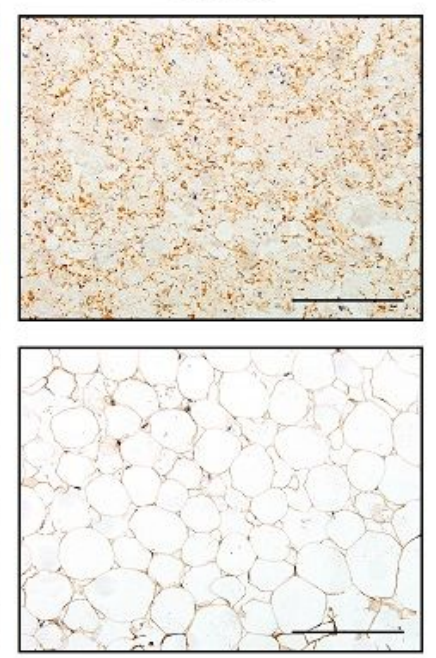

B

Laminin

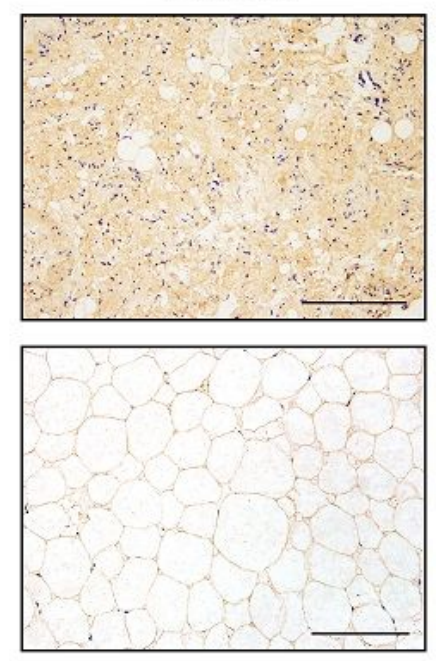

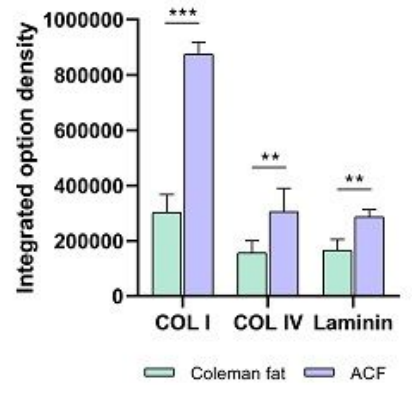

\section{Figure 4}

Collagen components assessment of ACF. a Immunohistochemical staining of COL I, COL IV, and laminin in ACF. $d$ Semi-quantitative analysis of COL I, COL IV, and laminin in ACF. Scale bar $=100 \mu \mathrm{m} .{ }^{*} p<0.05$, $\star \star p p 0.01, * \star * p<0.001$. 

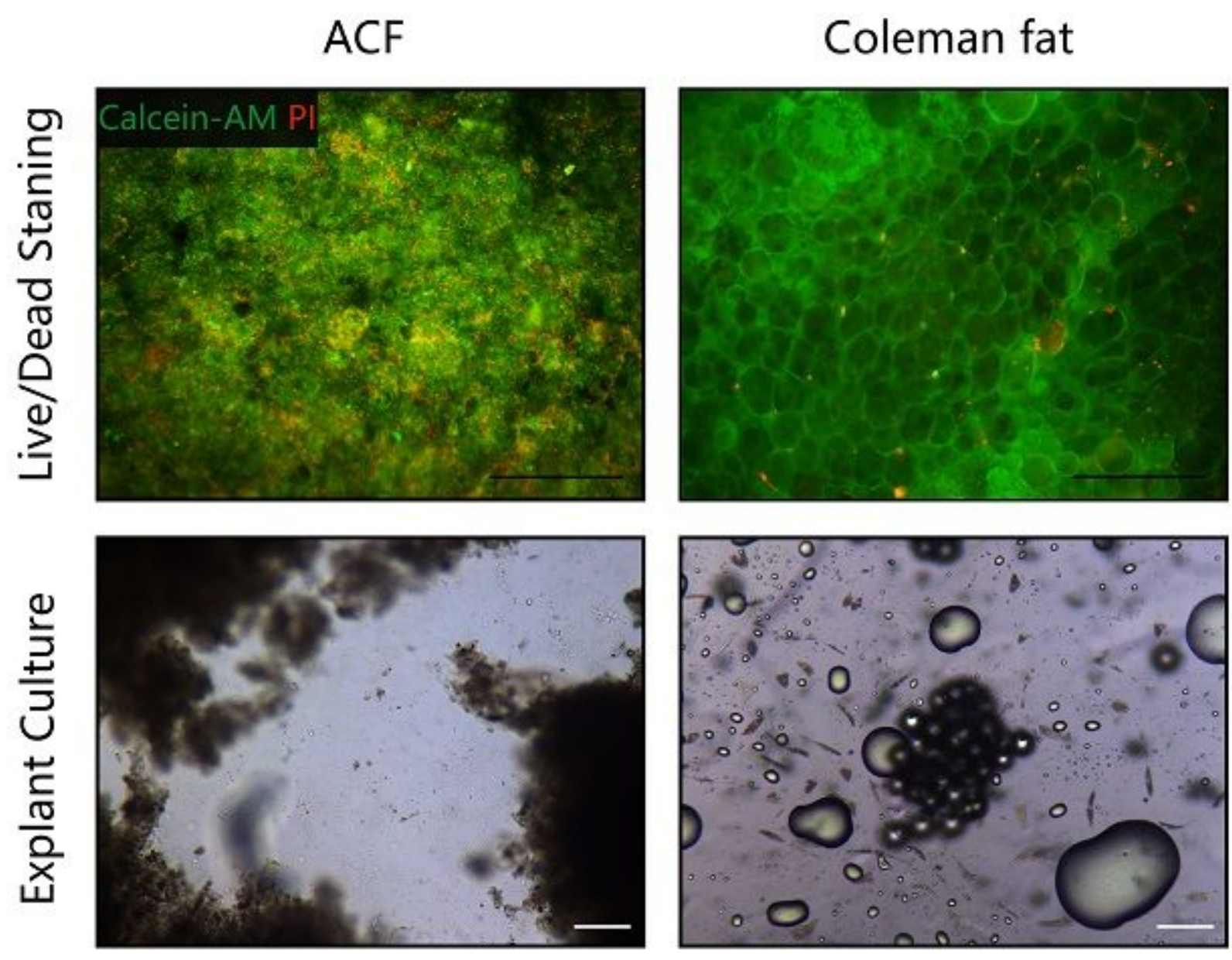

Figure 5

Cell viability assessment of ACF and Coleman fat. (Above) Live/dead staining of ACF and Coleman fat. Scale bar $=100 \mu \mathrm{m}$. (Below) Explant culture assays of ACF and Coleman fat. Scale bar $=200 \mu \mathrm{m}$. 
A

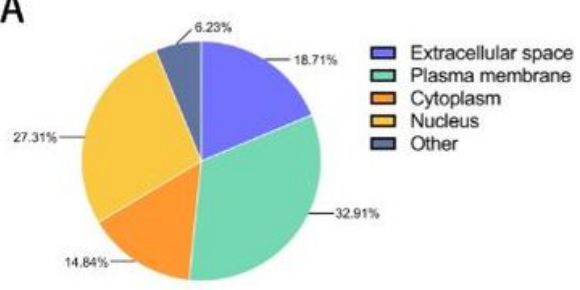

B

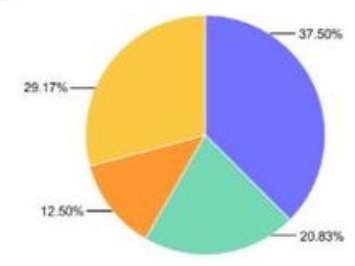

C

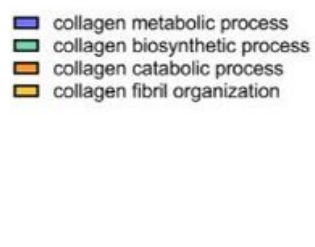

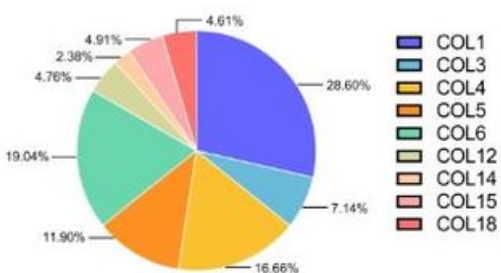

D

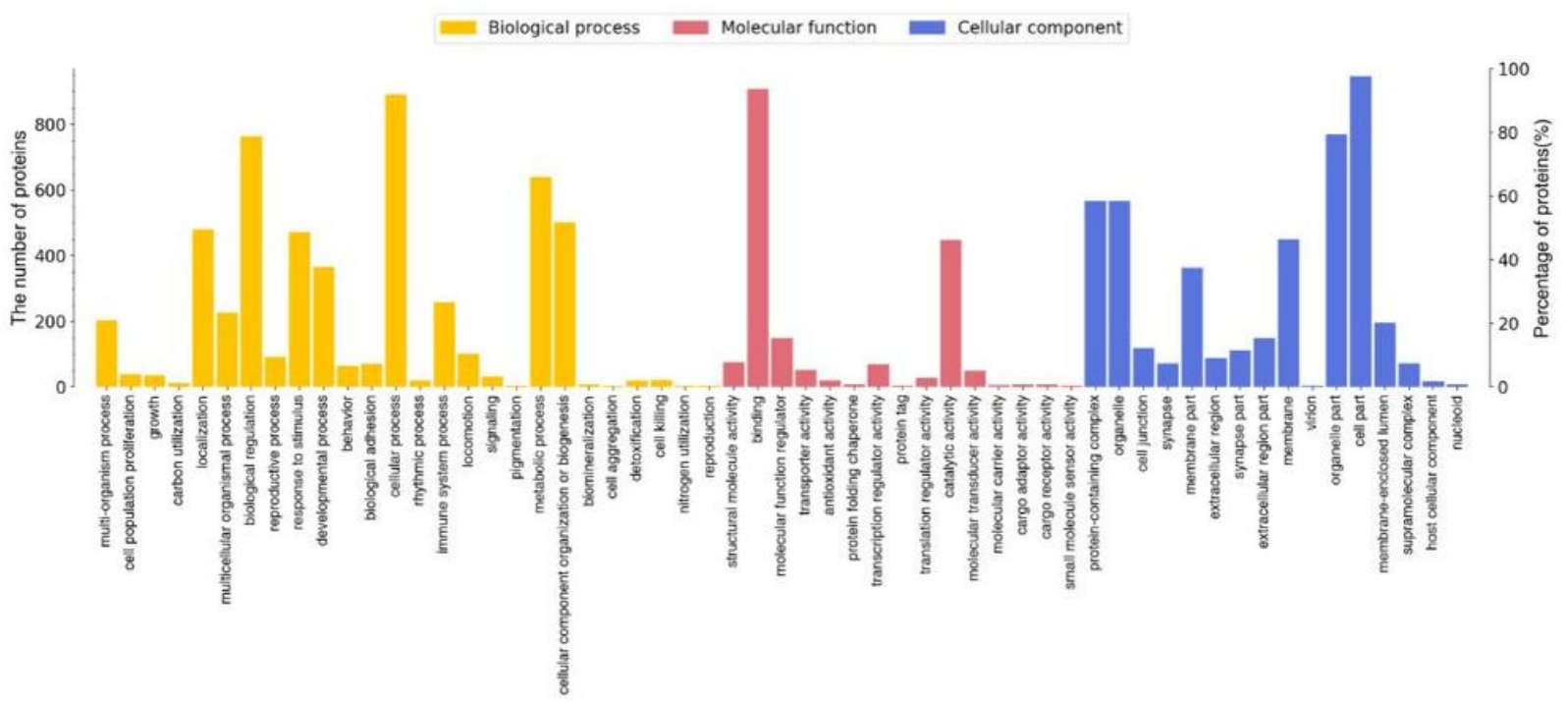

Figure 6

Mass spectrometry-based quantitative proteomic analysis of ACF and classification of identified proteins. a Subcellular distribution of proteins in ACF. b Distribution of proteins involved in the biological process of collagen in ACF. c Distribution of collagen types in ACF. $d$ Biological processes and molecular function and cellular components categories of identified proteins in ACF 

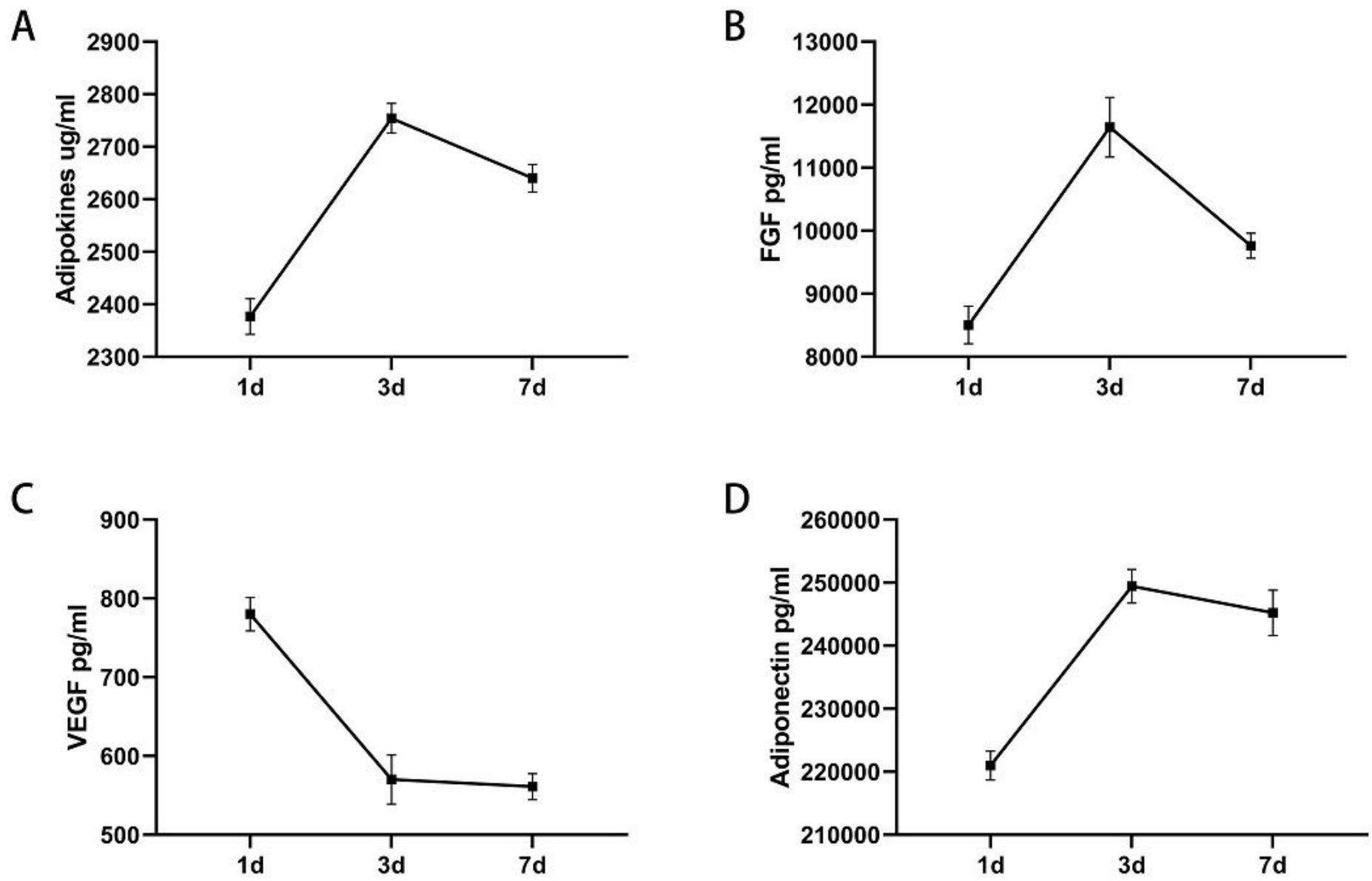

\section{Figure 7}

Adipokines release of ACF in vitro. a Concentrations of total adipokines released from ACF on days 1, 3 and 7. b,c,d Concentrations of FGF, VEGF and adiponectin released from ACF on days 1, 3 and 7. 


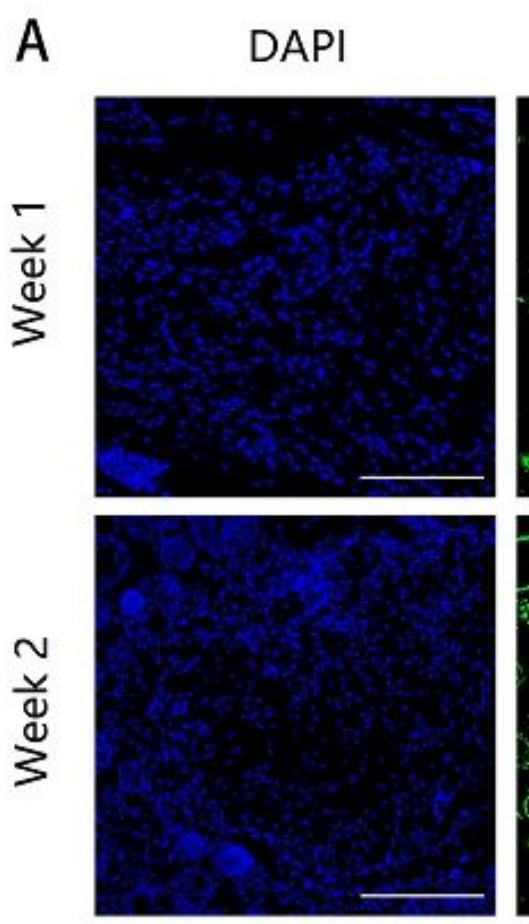

Procollagen
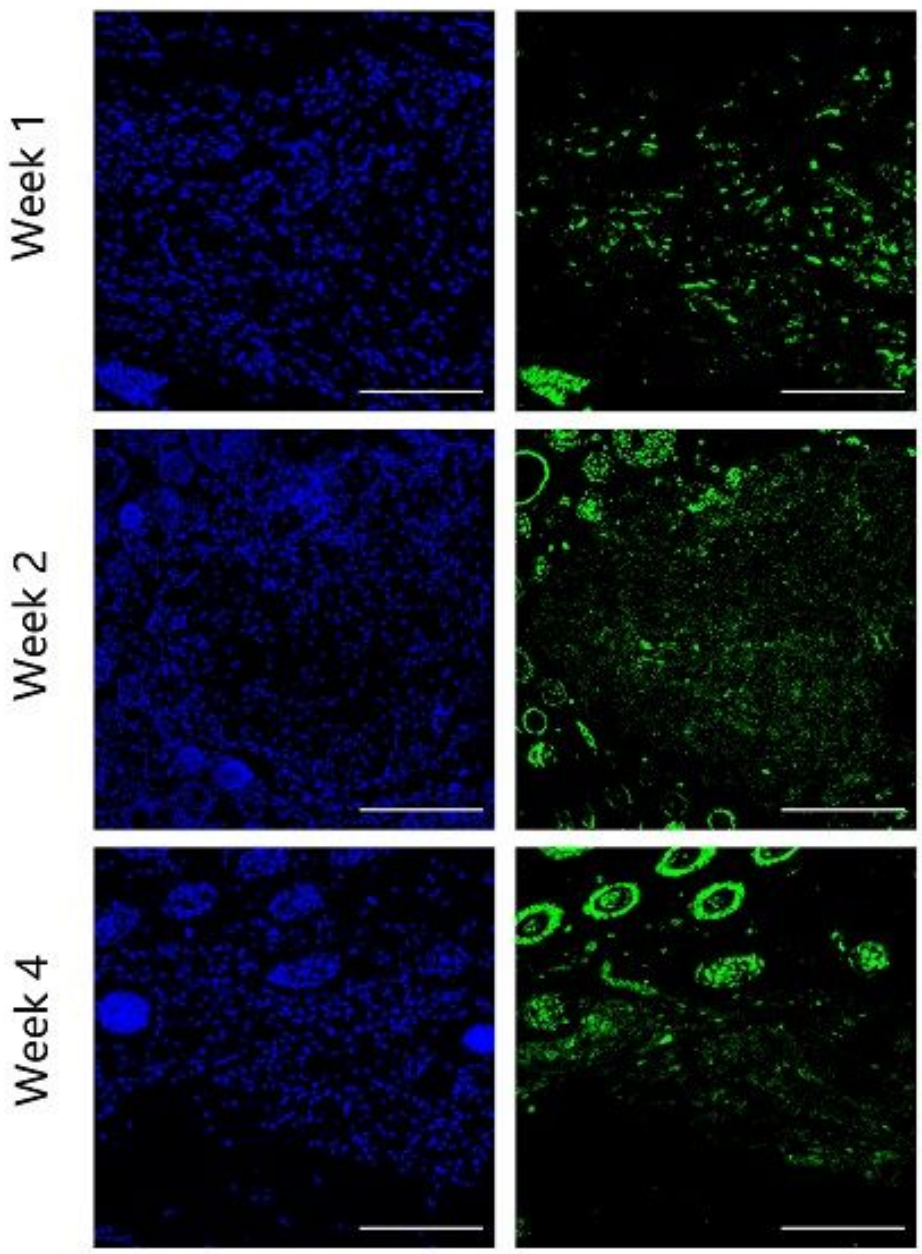

B

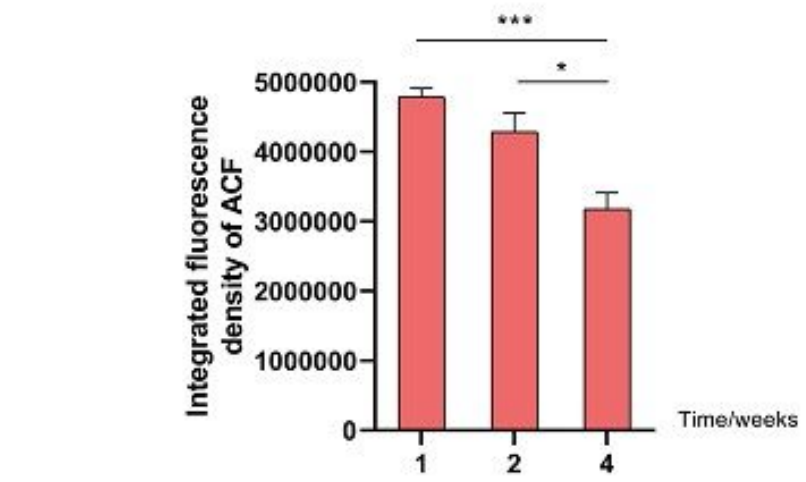

ACF
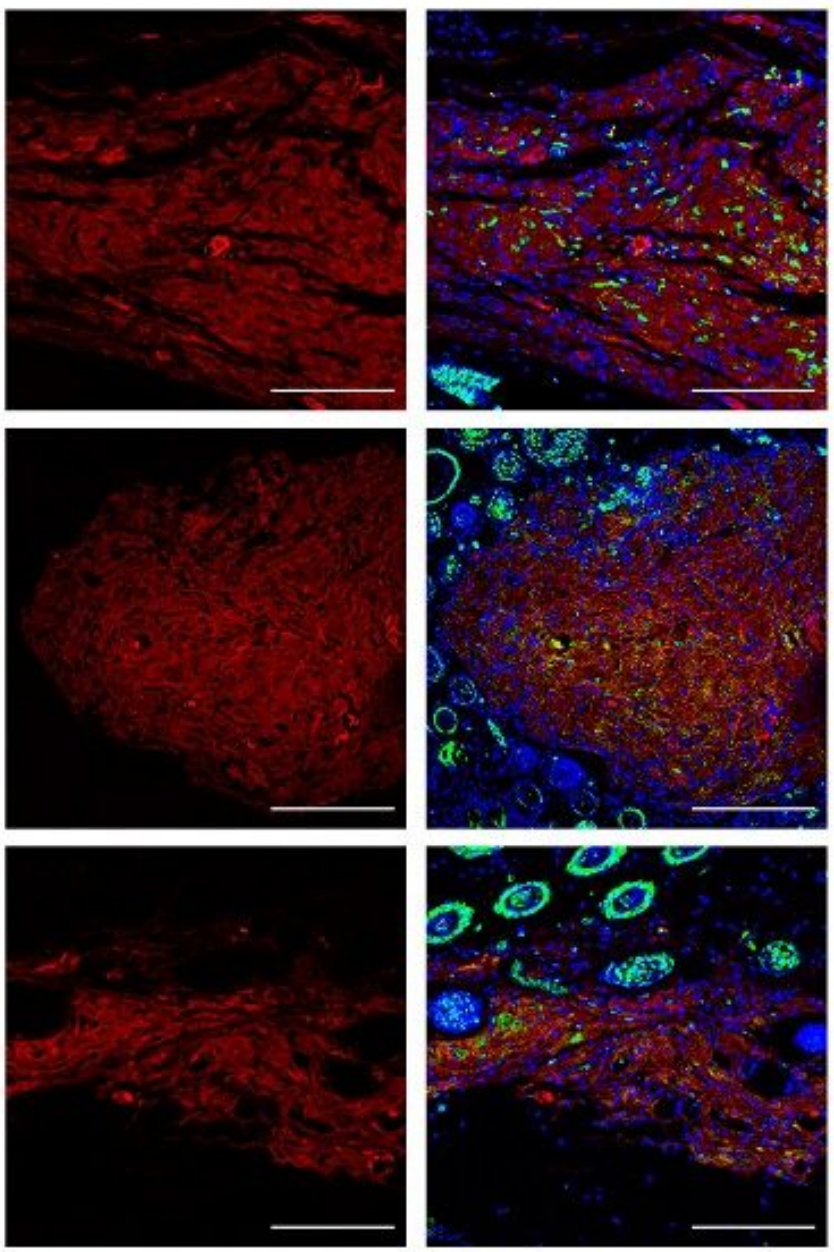

(6)

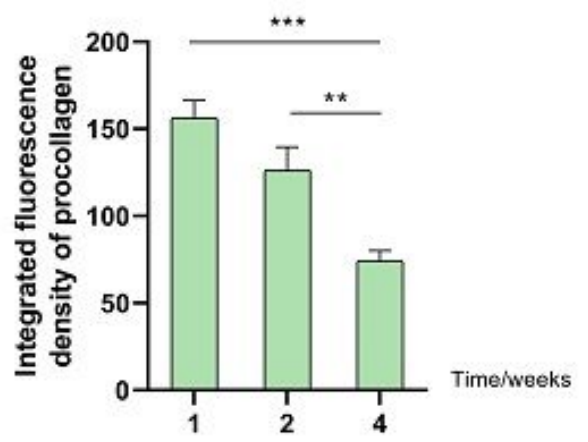

\section{Figure 8}

Histological evaluation of ACF in vivo remodeling process. a Immunofluorescence staining of AF647 labeled-ACF and procollagen. $b$ Semi-quantitative analysis of ACF retention and procollagen expression in ACF. Scale bar $=200 \mu \mathrm{m} .{ }^{\star} \mathrm{p}<0.05,{ }^{\star \star} \mathrm{p}<0.01,{ }^{\star \star \star} \mathrm{p}<0.001$. 
A
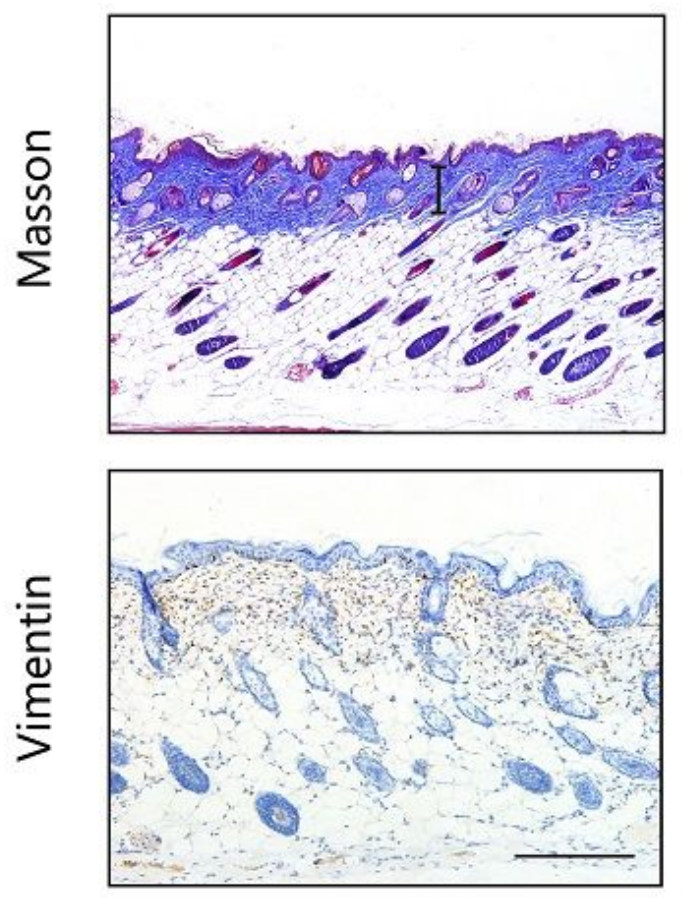

ACF-terated
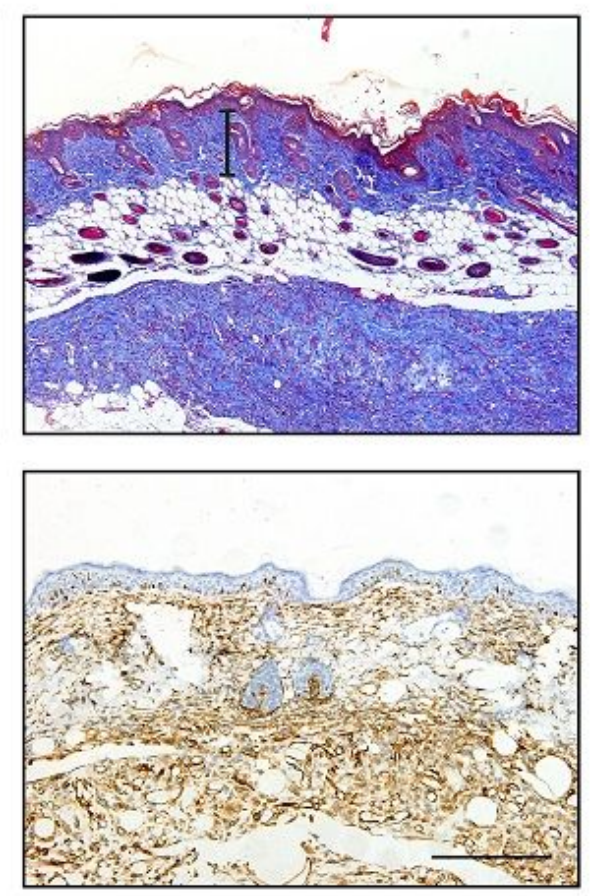

B
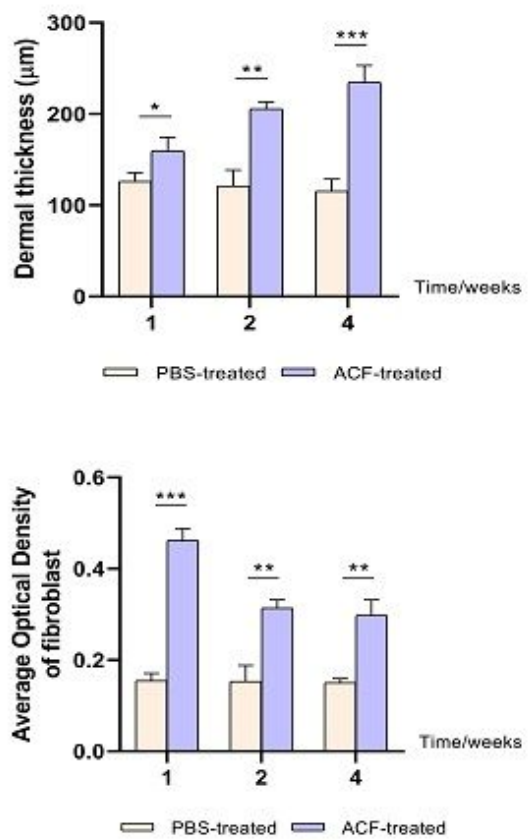

\section{Figure 9}

Masson staining and the fibroblast expression of mouse skin samples. a Dermal thickness and the fibroblast expression of both groups at week 1 . The arrows indicate the dermal portion of the skin. $b$ Semi-quantitative analysis of the dermal thickness of both groups at all time points. c Semi-quantitative analysis of the fibroblast expression in the dermis of both groups at all time points. Scale bar $=200 \mu \mathrm{m}$. ${ }^{*} \mathrm{p}<0.05,{ }^{\star *} \mathrm{p}<0.01,{ }^{\star \star *} \mathrm{p}<0.001$. 

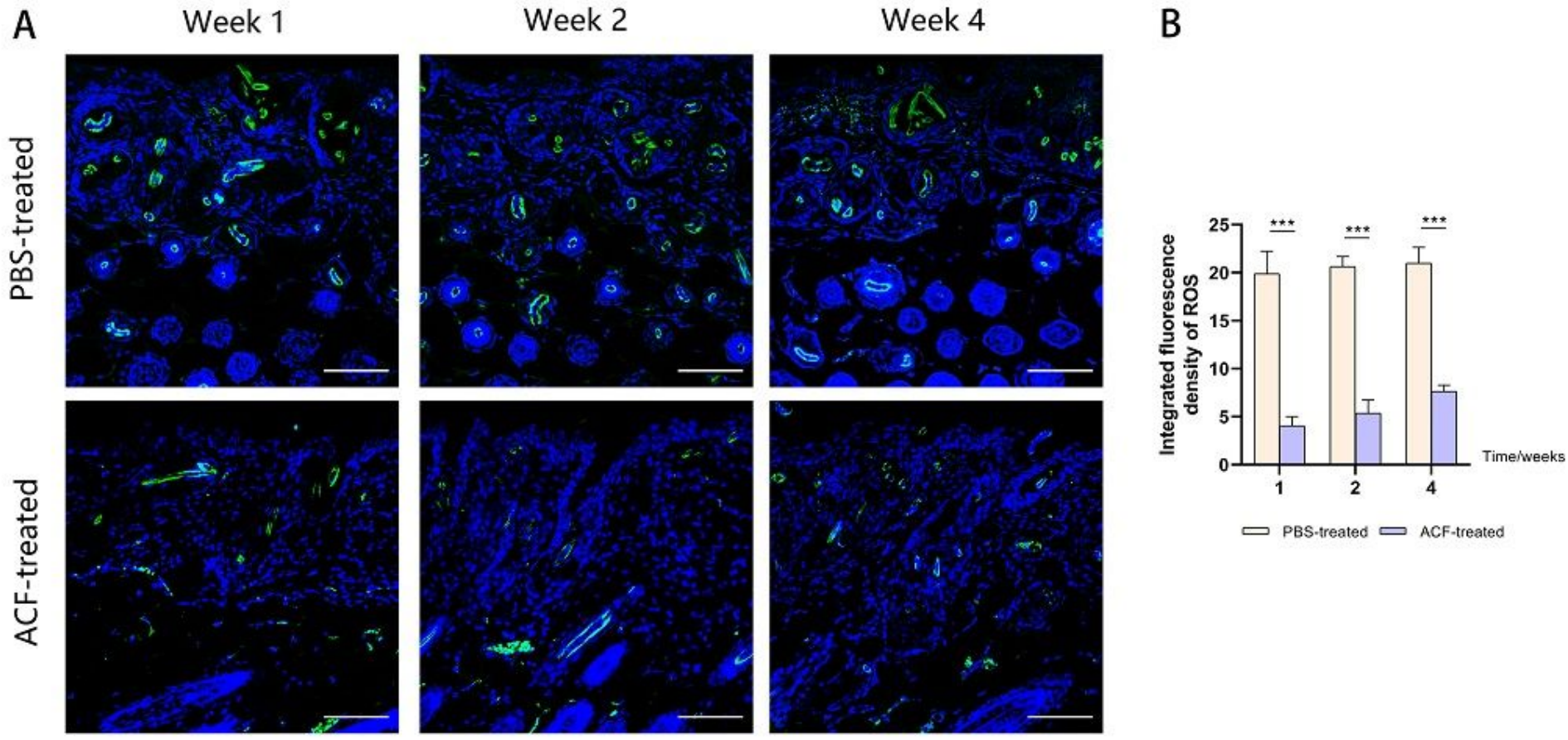

Figure 10

ROS production of mouse skin samples of ACF-treated group and control group. a ROS production evaluated by dihydroethidium staining of both groups. b Semi-quantitative analysis of the ROS production in the dermis of both groups. Scale bar $=200 \mu \mathrm{m} .{ }^{*} \mathrm{p}<0.05,{ }^{\star \star} \mathrm{p}<0.01,{ }^{\star \star \star} \mathrm{p}<0.001$.
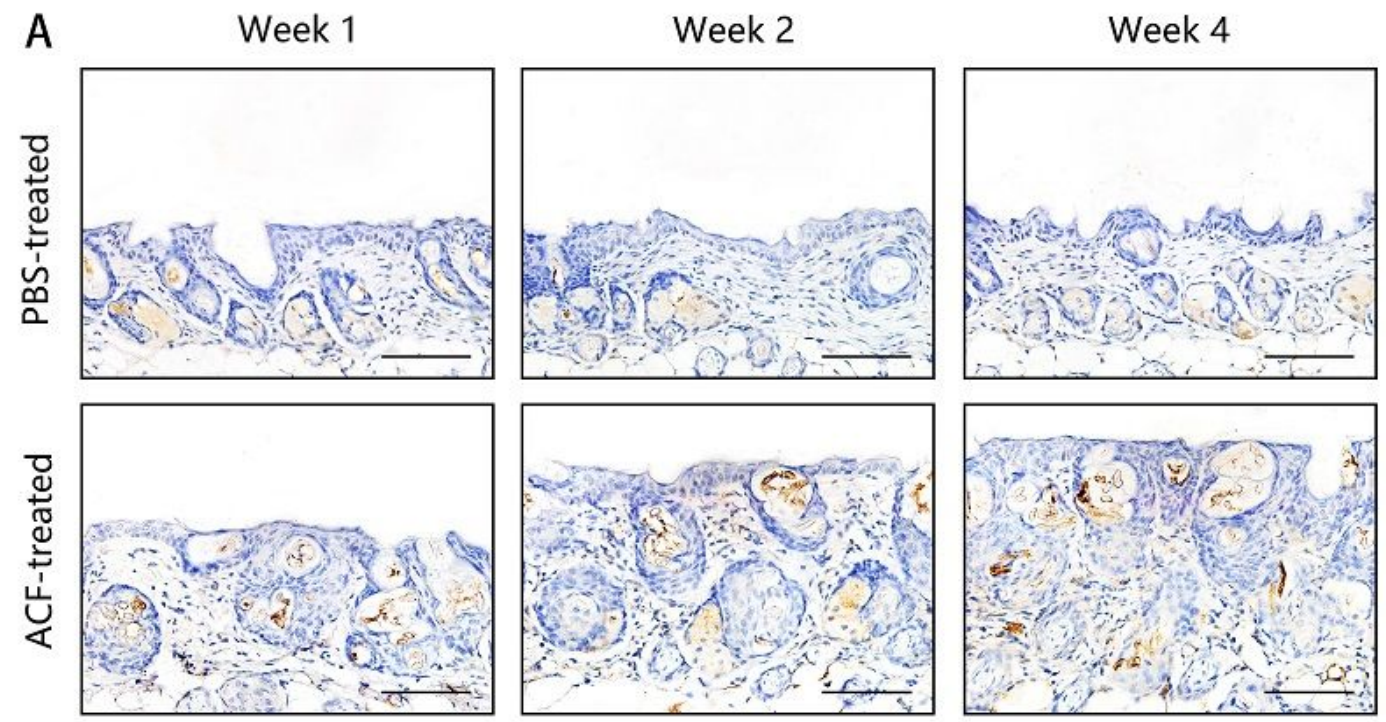

B
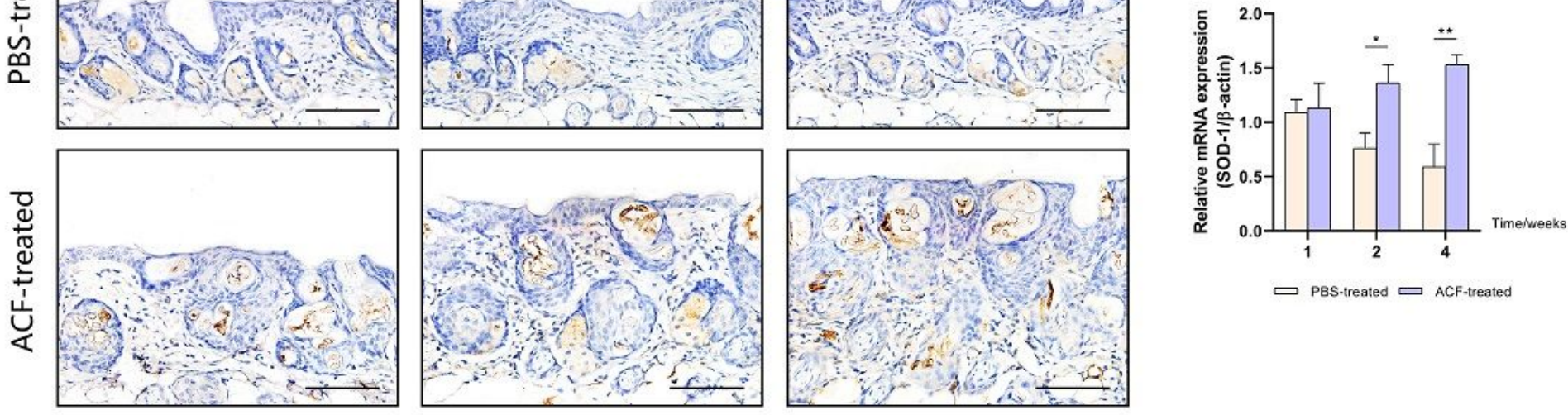

Figure 11 
The expression of SOD-1 of mouse skin samples of ACF-treated group and control group. a The expression of SOD-1 in the dermis of both groups. b,c,d Quantitative reverse transcription PCR analysis of the SOD-1 expression in the dermis of both groups. Scale bar $=100 \mu \mathrm{m} .{ }^{*} \mathrm{p}<0.05,{ }^{\star \star} \mathrm{p}<0.01,{ }^{\star \star \star} \mathrm{p}<$ 0.001 .
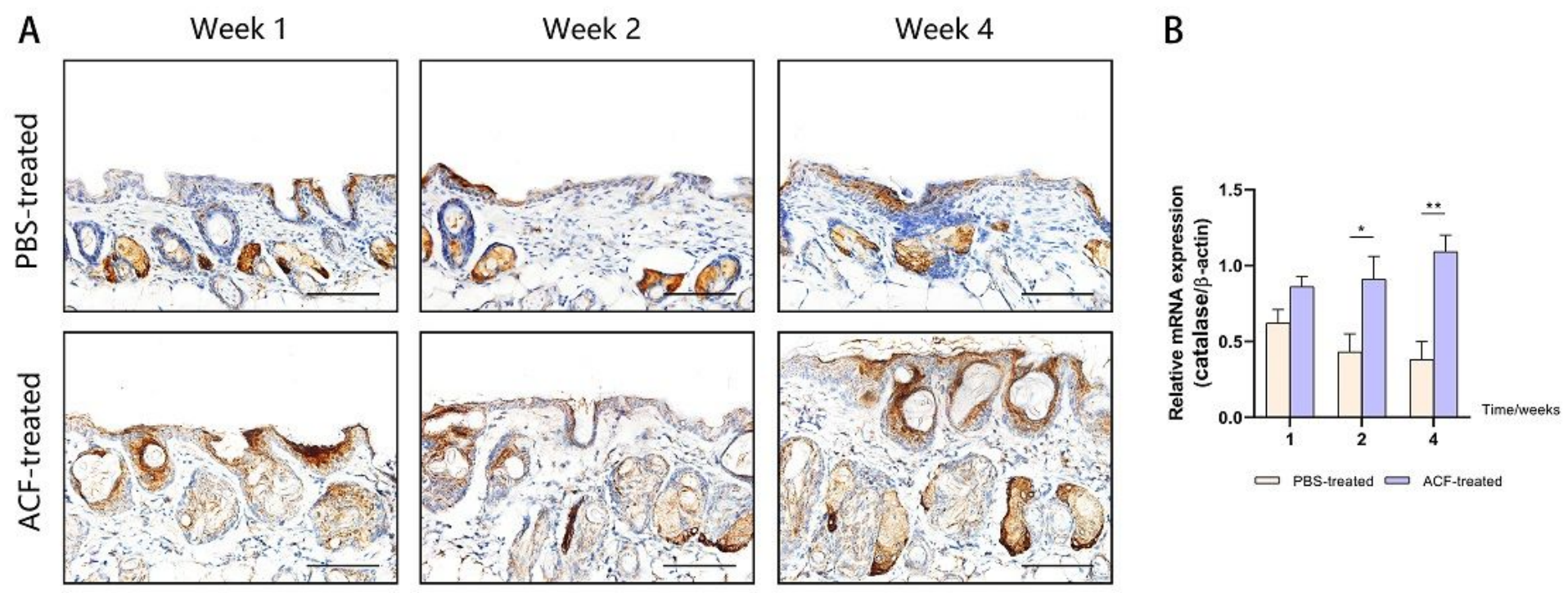

\section{Figure 12}

The expression of catalase of mouse skin samples of ACF-treated group and control group. a The expression of catalase in the dermis of both groups. b,c,d Quantitative reverse transcription PCR analysis of the catalase expression in the dermis of both groups. Scale bar $=100 \mu \mathrm{m} .{ }^{*} \mathrm{p}<0.05,{ }^{\star \star} \mathrm{p}<0.01,{ }^{\star \star \star} \mathrm{p}<$ 0.001 .

A Week 1
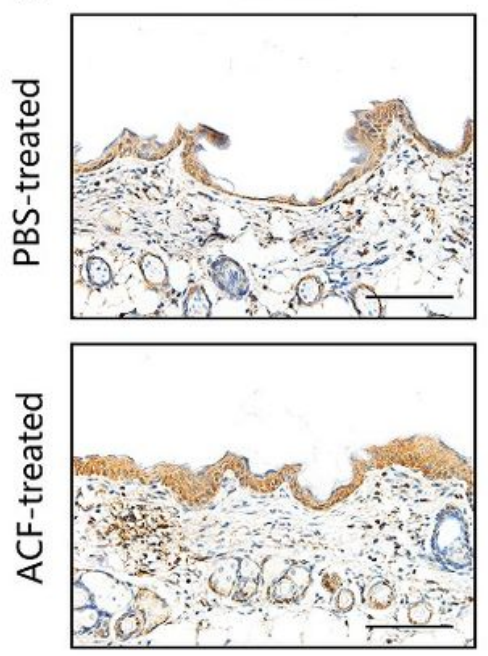

Week 2
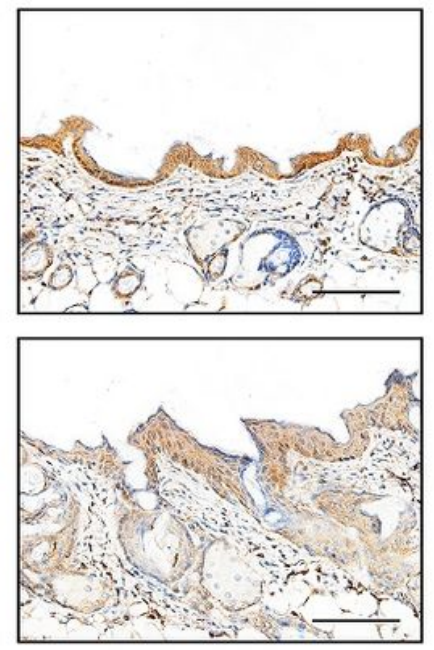

Week 4

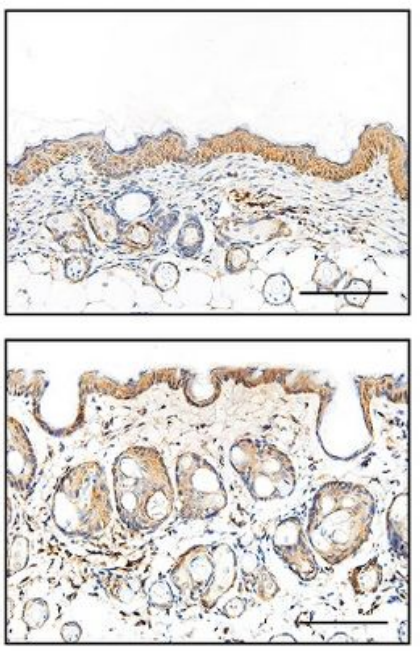

B

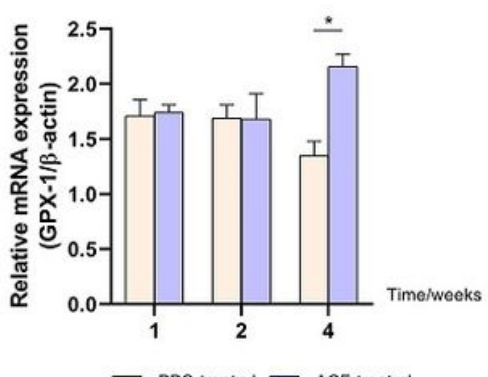

Figure 13 
The expression of GPX-1 of mouse skin samples of ACF-treated group and control group. a The expression of GPX-1 in the dermis of both groups. b,c,d Quantitative reverse transcription PCR analysis of the GPX-1 expression in the dermis of both groups. Scale bar $=100 \mu \mathrm{m}$. ${ }^{\star} p<0.05,{ }^{\star \star} p<0.01,{ }^{\star \star \star} p<$ 0.001 .
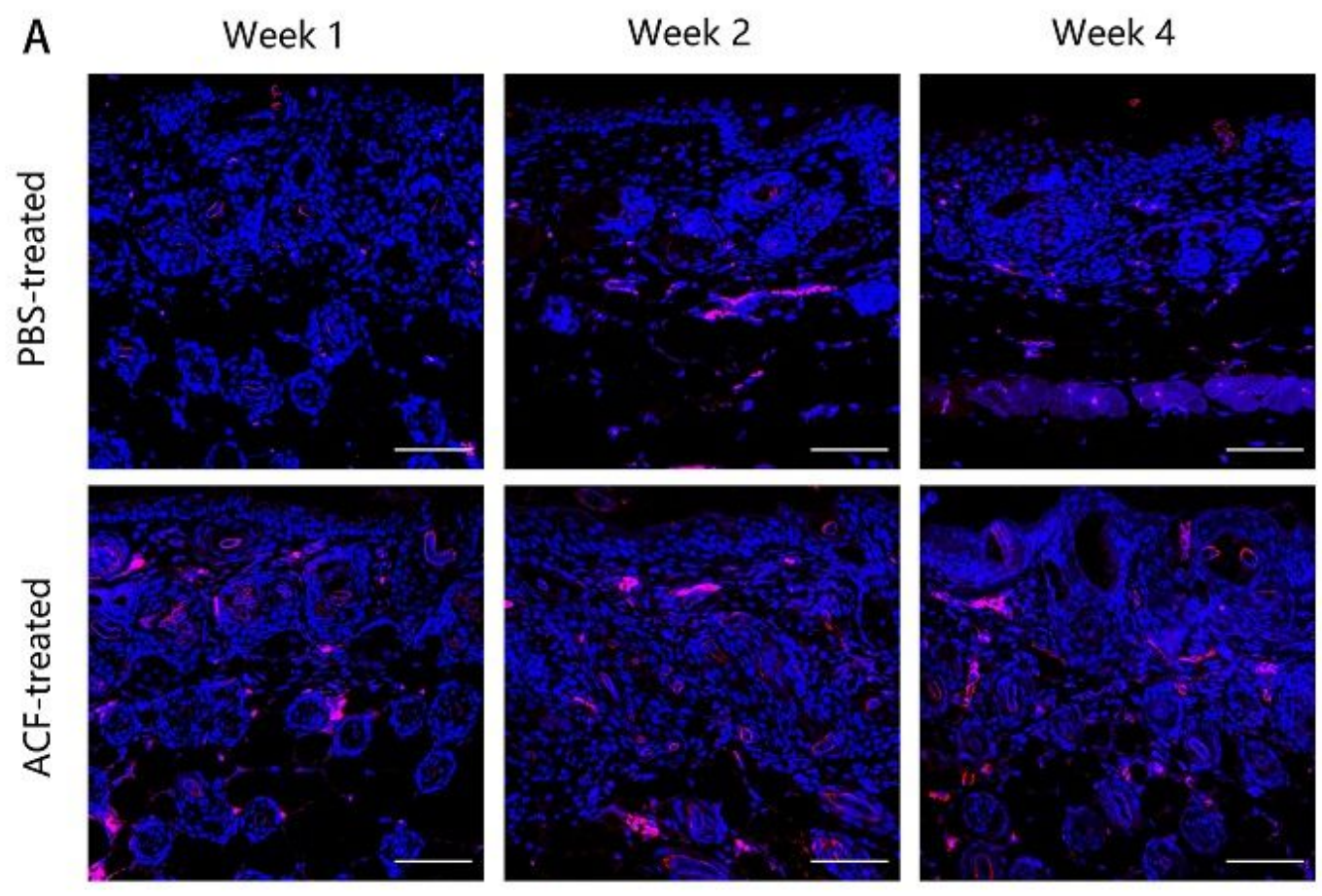

B

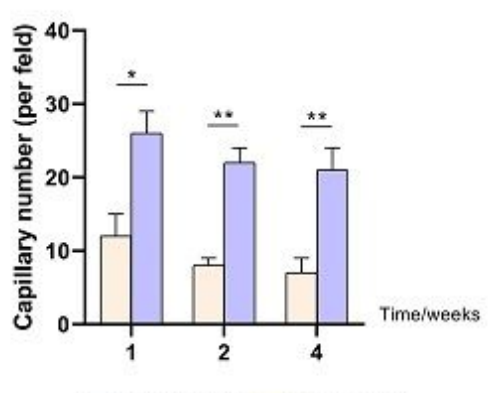

$\square$ PBS-treated $\square$ ACF-treated

\section{Figure 14}

Angiogenesis in mouse skin samples of ACF-treated group and control group. a Immunostaining of CD31+ vessels in the dermis of both groups. b Semi-quantitative analysis of CD31+vessels in the dermis of both groups. Scale bar $=200 \mu \mathrm{m} .{ }^{*} p<0.05,{ }^{* \star} p<0.01,{ }^{\star \star *} p<0.001$. 


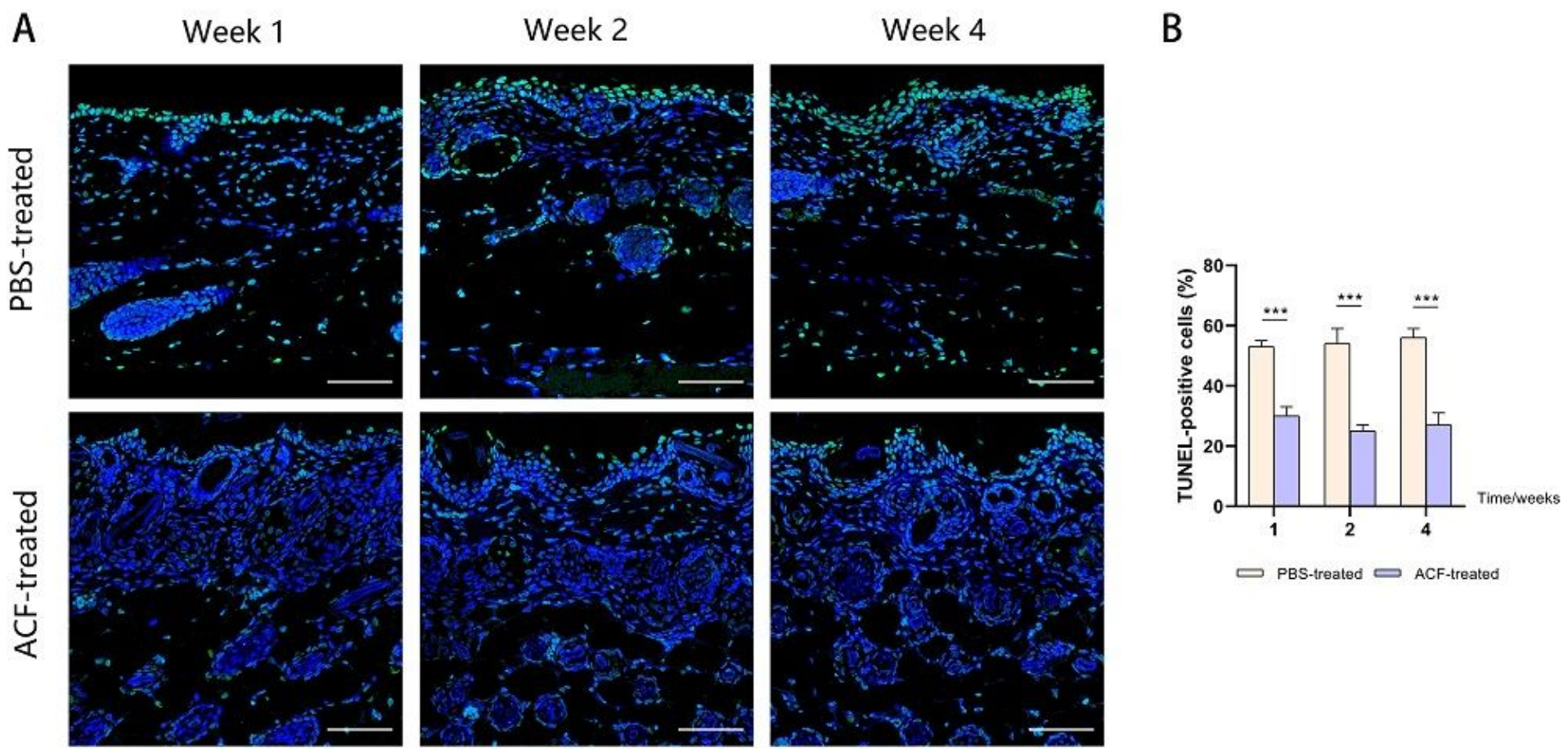

Figure 15

Cell apoptosis in mouse skin samples of ACF-treated group. a Immunostaining of apoptotic cells following labeling with a TUNEL staining kit in the dermis of both groups. $b$ Semi-quantitative analysis of apoptotic cells in the dermis of both groups. Scale bar $=200 \mu \mathrm{m} .{ }^{\star} p<0.05,{ }^{\star \star} \mathrm{p}<0.01,{ }^{\star \star \star} \mathrm{p}<0.001$.

\section{Supplementary Files}

This is a list of supplementary files associated with this preprint. Click to download.

- Additionalfile1..jpg

- Additionalfile2video.mp4

- Table1.Distributionofproteins.jpg 